\title{
Effect of the type of siliceous template and carbon precursor on physicochemical and catalytic properties of mesoporous nanostructured carbon-palladium systems
}

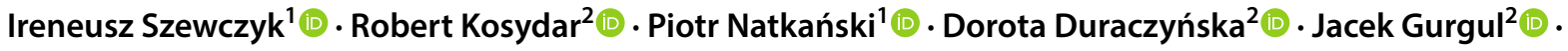 \\ Piotr Kuśtrowski ${ }^{1}$ (D) Alicja Drelinkiewicz ${ }^{2}$ D
}

Published online: 24 May 2020

(C) The Author(s) 2020

\begin{abstract}
Two series of nanostructured carbon replicas were synthesized through a hard-templating method using ordered SBA-15 or disordered mesoporous spherical silica gel as the templates and furfuryl alcohol or sucrose as the carbon source. They were explored to synthesize the Pd catalysts (ca. $1.5 \mathrm{wt} \%$ Pd loading) by the colloid-based microemulsion procedure which allowed preparing the catalysts of monodispersed Pd particles of similar size (4-5 nm). The palladium phase in the catalysts (e.g. the Pd particles distribution and extent of agglomeration) varied depending on the textural and surface characteristics of the carbon replicas. In the furfural hydrogenation studied as a probe reaction (2-propanol, $35^{\circ} \mathrm{C}, 6$ bar $\mathrm{H}_{2}$ ), furfuryl alcohol and tetrahydrofurfuryl alcohol were the major products formed. The catalytic reactivity has been related to the properties of the $\mathrm{Pd}$ phase including the surface Pd concentration and the extent of the Pd particles aggregation accompanied by the nearest palladium environment expressed by the surface $\mathrm{O} / \mathrm{Pd}$ atomic ratio. At high $\mathrm{O} / \mathrm{Pd}$ ratio the reaction slowed down because of a facilitated furfuryl alcohol adsorption resulting most probably in a blockage of the active sites. $\mathrm{The}^{\mathrm{SiO}} \mathrm{Sucrose} \mathrm{system}$ provided the catalyst which was characterized by relatively low $\mathrm{O} / \mathrm{Pd}$ ratio with well-dispersed Pd particles and the highest and stable activity for the furfural hydrogenation. The SBA-series catalysts, with lower O/Pd and more aggregated Pd particles, exhibited lower activity but somewhat higher tendency to the tetrahydrofurfuryl alcohol formation.
\end{abstract}

Keywords Ordered mesoporous carbon $\cdot$ Pd nanoparticles $\cdot$ Oxygenated carbon functionalities $\cdot$ Furfural hydrogenation

\section{Introduction}

Electronic supplementary material The online version of this article (https://doi.org/10.1007/s10934-020-00896-y) contains supplementary material, which is available to authorized users.

Piotr Kuśtrowski

piotr.kustrowski@uj.edu.pl

$\triangle$ Alicja Drelinkiewicz

ncdrelin@cyf-kr.edu.pl

Ireneusz Szewczyk

ireneusz.szewczyk@doctoral.uj.edu.pl

Robert Kosydar

nckosyda@cyf-kr.edu.pl

Piotr Natkański

piotr.natkanski@uj.edu.pl
Carbonaceous materials have been widely used as supports of metal-based catalysts under various forms: activated carbon, carbon black, multiwall carbon nanotubes, nanostructured carbon replicas (OMC) synthesized by templating methods $[1,2]$. The OMC carbons are of great interest due to the regular array of uniform pores, high specific surface

\author{
Dorota Duraczyńska \\ ncduracz@cyf-kr.edu.pl \\ Jacek Gurgul \\ ncgurgul@cyf-kr.edu.pl \\ 1 Faculty of Chemistry, Jagiellonian University, Gronostajowa \\ 2, 30-387 Krakow, Poland \\ 2 Jerzy Haber Institute of Catalysis and Surface Chemistry, \\ Polish Academy of Sciences, Niezapominajek 8, \\ 30-239 Krakow, Poland
}


area and large pore volume $[2,3]$. They consist of a network of interconnected regular carbon rods forming porous structure with predomination of mesopores between the rods and small contribution of micropores-defects in the walls of the carbon rods. The structural properties of OMC, including the porous structure and pore width are mainly influenced by template (MCM-48, SBA-15, HMS), whereas the carbon precursor (sucrose, furfuryl alcohol, anthracene) affects specific surface area, graphitization degree, and surface properties.

The CMK-3 carbon obtained with SBA-15 template and sucrose precursor was the most frequently applied as support for the noble metal-containing catalysts studied in electrocatalytic [4] and catalytic reactions [5-9]. For instance, the Ru/CMK-3 catalysts were very effective for ammonia decomposition [5] and nitrobenzene hydrogenation [6, 7], the Pt/CMK-3 catalysed enantioselective hydrogenation of $\alpha$-ketoesters [8] and benzaldehyde hydrogenation [9]. However, the preparation of OMC-based catalysts with well-dispersed metal nanoparticles by conventional impregnation/ reduction method is difficult. The carbonization process eliminates most of oxygenated carbon groups which are the anchoring and nucleating sites for the metal precursor [10-14]. This implies an uncontrolled growth of the metal crystallites of size within a wide range. They are located not only outside of carbon rods but also between adjacent arrays $[15,16]$. Therefore more complicated procedures involving polyol [17] or carbonization of a mixture consisting of the metal ions $\left(\mathrm{H}_{2} \mathrm{PtCl}_{6}\right.$, acetylacetonate of $\left.\mathrm{Pt}, \mathrm{Ru}\right)$ and carbon precursor (sucrose) inside the pores of SBA-15 template have been tested [17-19].

The nanostructured carbon replicas were also synthesized using disordered templates, such as $\mathrm{MgO}$ [20], $\mathrm{CaCO}_{3}$ [21], $\mathrm{TiO}_{2}$ [22], natural clay minerals [23], silica [24] and $\gamma$-alumina [24-28]. Among them, the mesoporous carbon synthesized using sucrose precursor and silica template seems to be very attractive for catalytic application due to very high surface area (up to $2000 \mathrm{~m}^{2} / \mathrm{g}$ ) and high porosity (ca. $2 \mathrm{~cm}^{3} / \mathrm{g}$ ) with domination of uniform mesopores ca. 10 $\mathrm{nm}$ width and only a trace of micropores [28]. This structure was a result of small and thin carbon sheets which joined each other forming a rigid framework [25, 26]. Its counterpart synthesized using furfuryl alcohol precursor was characterized by a somewhat lower surface area [24].

This synthesis procedure is adopted in the present work in order to prepare the mesoporous carbons of potential application as supports for the Pd catalysts. Thus, using disordered mesoporous silica gel as the template and sucrose (ss) or furfuryl alcohol (ff) as the carbon source, the first series of carbon replicas denoted as $\mathrm{C}(\mathrm{ss}-\mathrm{Si})$ and $\mathrm{C}(\mathrm{ff}-\mathrm{Si})$ are synthesized. The second series includes the carbon replicas denoted as $\mathrm{C}$ (ss-SBA) and $\mathrm{C}$ (ff-SBA) prepared using the same carbon sources but the ordered nanostructured SBA-15 as the template. These carbon samples are applied as the supports for palladium catalysts. The hydrogenation of furfural (FU) is used as a probe reaction to compare catalytic properties of the Pd phase in the synthesized catalysts [29].

The palladium was incorporated by means of colloidbased "water-in-oil" microemulsion method (ME). According to the ME procedure, the palladium particles are first prepared in the emulsion medium and then deposited on the support. Metal particles are formed by chemical reduction (by $\mathrm{NaBH}_{4}, \mathrm{~N}_{2} \mathrm{H}_{4}$ ) of metal precursors within aqueous droplets. Thus, a growth of the metal particles is restricted by the surfactant thus promoting monodispersed metal particles [30-33]. Metal particles are transferred onto a support by destabilization of the colloidal dispersion carried out in the presence of the support by slow addition of tetrahydrofuran (THF) [34]. The chemical compatibility between the support surface and hydrophobous "oil phase" of microemulsion (cyclohexane, isooctane) influences the metal particles distribution in the final catalyst as it determines the wetting of support by liquid. Therefore, hydrophobic supports, like carbons, allowing more effective surface wetting, are preferred $[35,36]$.

Palladium was chosen taking into account its well-known high activity for the hydrogenation of reagents with $\mathrm{C}=\mathrm{C}$ and $\mathrm{C}=\mathrm{O}$ bonds, among them furfural. Furfural is an important platform reagent for lignocellulosic biofuels and valueadded chemicals which can be obtained via catalytic hydrogenation (Scheme 1). These include 2-methylfuran (2-MF) and 2-methyltetrahydrofuran, furfuryl alcohol (FA) and tetrahydrofurfuryl alcohol (THFA), furan (FUR), tetrahydrofuran (THF), as well as various cyclo-products (cyclopentanone) and aliphatic diols [29]. The hydrogenation can proceed efficiently both in the liquid and vapour phase. The former system is preferred for compatibility with the production of furfural via catalytic dehydration of hemicellulose. The catalysts containing noble $(\mathrm{Pt}, \mathrm{Pd}, \mathrm{Ru}, \mathrm{Rh})$ or non-noble $(\mathrm{Cu}, \mathrm{Ni}, \mathrm{Co}, \mathrm{Fe})$ metals $[29,37-39]$ as well as metaloxides $\left(\mathrm{ZrO}_{2}\right)[40]$ have been developed. Typically, the noble-metals based catalysts exhibit better activity (higher intrinsic hydrogenation activity) but they suffer from high cost of precious elements. Non-noble metals are more abundant and cheaper. However, frequently the combinations with other metals or the choice of proper support, solvent are required to improve their activity, selectivity and stability. In the case of nonnoble metals, the application of high reaction temperature (150-200 ${ }^{\circ} \mathrm{C}$ ) and high $\mathrm{H}_{2}$ pressure $(0.2-0.5 \mathrm{MPa})$ is needed [29]. These reaction conditions could be hazardous to our $\mathrm{Pd} / \mathrm{C}$ catalysts. Some changes in the catalysts surface such as the reduction of oxygenated carbon groups and the metal particles migration/aggregation might proceed similarly to previous results for the carbon-supported $\mathrm{Pd}$, Pt catalysts [41, 42]. On the other hand, an easy dissociation of hydrogen by Pd resulted in effective FU hydrogenation under mild 
Scheme 1 Selected reaction pathways during the hydrogenation of furfural (FU) in 2-propanol; ( $F A$-furfuryl alcohol, THFA — tetrahydrofurfuryl alcohol, 2-MF-2-methylfuran, FUR - furan, $T H F$ - tetrahydrofuran, $T H F U$ - tetrahydrofurfural, $E T$ - furfuryl isopropyl ether, $A C$-furfural diisopropyl acetal)

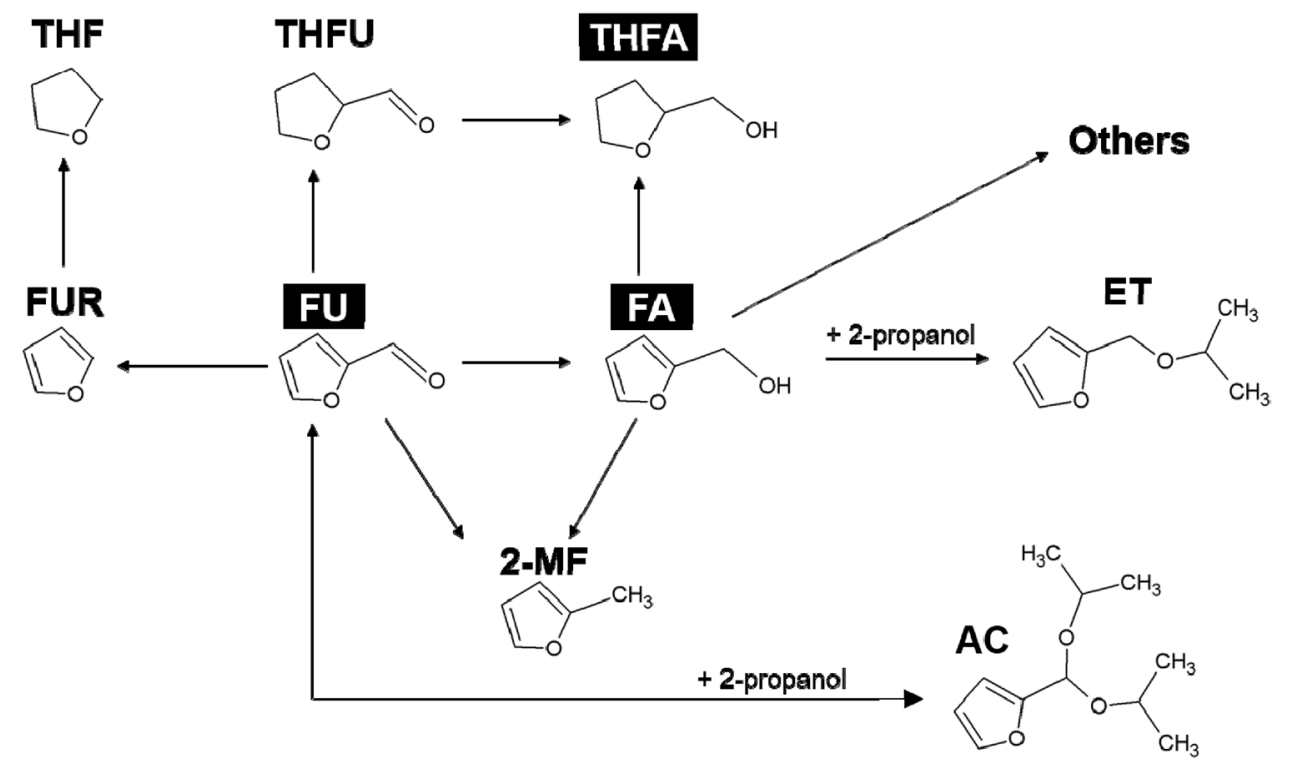

conditions (room temperature and $\mathrm{H}_{2}$ pressure of 1-3 bar) [43-45].

In general, the adsorption configuration of FU molecule, which is highly dependent on the nature of the metal, determines to a large extent the hydrogenation pathway and the products distribution. The interaction of $\mathrm{FU}$ with a $\mathrm{Cu}$ surface involves the $\eta^{1}-(\mathrm{O})$ adsorption mode via the aldehyde group. The strong repulsion of the furan ring with the $\mathrm{Cu}$-surface limits the hydrogenation of the furan ring. As a result, the $\mathrm{Cu}$-based catalysts are a choice for the selective formation of furfuryl alcohol which is the most important product to date. The copper chromite catalyst is usually employed in the industrial applications for the FA production. However, this process poses a serious drawback caused by the toxic nature of $\mathrm{Cr}$ and requires harsh conditions (ca. $200{ }^{\circ} \mathrm{C}, 2-10 \mathrm{MPa}$ ). The $\mathrm{Cr}$-free $\mathrm{Cu}$-based catalysts like $\mathrm{Cu}$ doped porous metal oxides $\left(\mathrm{CuMgAlO}_{\mathrm{X}}\right)$ also exhibited very attractive performance. Moreover, the $\mathrm{Co}$, Fe-based catalysts also promoted the selectivity to the FA formation. Over the $\mathrm{Pd}, \mathrm{Pt}, \mathrm{Ni}$ metals, FU tends to adopt an $\eta^{2}-(\mathrm{C}, \mathrm{O})$ adsorption mode in which the furan ring lies flat onto the metal surface with both the $\mathrm{C}$ and $\mathrm{O}$ atoms of the carbonyl group bonded to the surface. The flat adsorption configuration has been associated with a strong interaction between the metal and the $\pi$ bonds in the furan ring, originating from an $\mathrm{sp}^{2}$ to $\mathrm{sp}^{3}$ rehybridization. On these metals, the reactions involve both the furan ring and the aldehyde group and the products of hydrogenation, hydrogenolysis, decarbonylation can be formed. The activity/selectivity aspects of $\mathrm{Cu}$ and other nonnoble metals and that of Pd are summarized in Table S1 in supplementary data.

The products selectivity over Pd-catalysts varied depending on the reaction system both in terms of catalyst (support properties, Pd particle size) as well as the reaction conditions (solvent, temperature, pressure) [45-49]. The furan ring hydrogenation producing overhydrogenated products (THFA, THF) was promoted by high $\mathrm{H}_{2}$ pressure, whereas high temperature promoted the hydrogenolysis and decarbonylation reactions producing 2-MF and furan. Accordingly, Nguen-Huy et al. [49] observed that in 2-propanol under 20 bar $\mathrm{H}_{2}$ at $180{ }^{\circ} \mathrm{C}$, the $\mathrm{Pd} / \mathrm{SiO}_{2}$ and $\mathrm{Pd} / \mathrm{Al}_{2} \mathrm{O}_{3}$ promoted the formation of $\mathrm{FA}$, whereas the $\mathrm{Pd}$ on carbon black preferred subsequent furan ring hydrogenation generating THFA. Alibali et al. [48] reported that in 2-propanol under mild reaction conditions $\left(30{ }^{\circ} \mathrm{C}, 3\right.$ bar $\mathrm{H}_{2}$ ) $\mathrm{Pd} / \mathrm{Al}_{2} \mathrm{O}_{3}$ and $\mathrm{Pd} / \mathrm{TiO}_{2}$ were much more selective towards THFA than Pd/activated carbon). Biradar et al. [46] obtained total hydrogenation of FU to THFA (2-propanol, $220^{\circ} \mathrm{C}, 3.4 \mathrm{MPa}_{2}$ ) employing the siliceous MFI-type molecular sieve-supported Pd. The selective transformation of FU to THFA has been related to the location of the Pd-particles in the cavities and channels of molecular size in the porous MFI structure. Recently, Chai et al. reported [50] a significant modulation of the FU hydrogenation selectivity employing the catalysts with the Pd nanoclusters encapsulated within MFI-type zeolites, silicate-1, Na-ZSM-5, H-ZSM-5. An influence of zeolite microenvironment resulted in the different reaction pathways (2-propanol, $175^{\circ} \mathrm{C}, 1 \mathrm{MPa}$ ). The Pd/H-ZSM-5 generated FA and THFA at similar selectivities (ca. 30\%), whereas over the Pd/Na-ZSM-5 catalyst the FA selectivity as high as $>90 \%$ was reported. In FU hydrogenation under nonconventional conditions using supercritical $\mathrm{CO}_{2}$ $\left(\mathrm{scCO}_{2}, 130^{\circ} \mathrm{C}, 8 \mathrm{MPa}\right)$ Liu et al. [51] obtained FA yield of $56 \%$ at FU conversion of $79 \%$ over Pd/activated carbon. Mironenko et al. [52] reported that the use of Pd/CNT and $\mathrm{Pd} / \mathrm{CB}$ (carbon black) catalysts in aqueous medium under mild conditions of $50{ }^{\circ} \mathrm{C}, 0.5-2 \mathrm{MPa}_{2}$ afforded 
FA yield $>90 \%$ at total FU conversion. At higher reaction temperature $\left(90^{\circ} \mathrm{C}\right)$ the FA yield decreased due to the formation of THFA over the Pd/CB with larger Pd particles.

In the present work the catalysts of similar Pd loading (1.1-1.4 wt\%) with nearly monodisperse Pd nanoparticles of ca. $4-5 \mathrm{~nm}$ in size are obtained using the ME method. The studies concentrate on the influence of porous structure and surface properties (oxygenated carbon groups) of the carbon replicas on the Pd phase including the metal particles penetration, distribution and extent of aggregation. The commercial carbon black (Vulcan XC-72) supported catalyst was also prepared by the ME method for the purpose of comparison. The aim of our experiments was to elucidate the effect exerted by the particular carbon support on the formation of Pd active sites for the furfural hydrogenation studied in 2-propanol solvent under mild reaction conditions $\left(35^{\circ} \mathrm{C}, 6\right.$ bar $\left.\mathrm{H}_{2}\right)$.

\section{Experimental}

\subsection{Preparation of carbon replicas}

SBA-15 synthesized according to the previously described procedure [53] and commercial mesoporous spherical silica gel (particle size 40-75 $\mu \mathrm{m}$, Sigma-Aldrich) were used as the templates. Furfuryl alcohol (Sigma-Aldrich) was deposited in the pore system of silica (C(ff-SBA) and $\mathrm{C}(\mathrm{ff}-\mathrm{Si}$ ) samples) by the precipitation polycondensation. The silica template $(3.0 \mathrm{~g})$ was added to an aqueous solution containing $6.0 \mathrm{~g}$ of FA dissolved in $91.0 \mathrm{~g}$ of water. Then, an $\mathrm{HCl}$ (Avantor) solution was introduced at $\mathrm{HCl} /$ FA molar ratio of 6 . The resulting slurry was heated up to $100{ }^{\circ} \mathrm{C}$ and left for $6 \mathrm{~h}$ under vigorous stirring. After cooling and separation by filtration, the isolated brown solid was washed thoroughly with water and dried overnight at room temperature.

The $\mathrm{C}(\mathrm{ss}-\mathrm{Si}$ ) and $\mathrm{C}$ (ss-SBA) carbon replicas were obtained using sucrose (Avantor) as the carbon source [54]. An aqueous solution containing $1.25 \mathrm{~g}$ of sucrose, $5.0 \mathrm{~g}$ of water and $0.14 \mathrm{~g}$ of sulfuric acid was introduced by the dry impregnation into the silica matrix $(10 \mathrm{~g})$. The obtained composites were dried in a furnace (first at $100{ }^{\circ} \mathrm{C}$ for $6 \mathrm{~h}$, and then at $160^{\circ} \mathrm{C}$ for $6 \mathrm{~h}$ ). The loading and drying procedures were subsequently repeated.

All synthesized materials were carbonized in a tubular furnace (heating rate of $1{ }^{\circ} \mathrm{C} / \mathrm{min}$, isothermal step at $850{ }^{\circ} \mathrm{C}$ for $4 \mathrm{~h}$ ) in a flow of nitrogen $\left(40 \mathrm{~cm}^{3} / \mathrm{min}\right)$. Removal of the silica templates was performed twice by a treatment with $5 \%$ $\mathrm{HF}$ (Avantor) at room temperature using $30 \mathrm{~cm}^{3}$ of the acid solution per $1 \mathrm{~g}$ of the composite, followed by washing with water and ethanol (96\%, Avantor).

\subsection{Preparation of palladium catalysts}

The catalysts were prepared by means of the microemulsion method (ME). The reverse micellar solutions were prepared using polyoxyethylene(7-8)octylphenyl ether (Triton X114) (Sigma-Aldrich) as the surfactant and cyclohexane (SigmaAldrich) as the "oil" phase. In all preparations parameter " $w$ " (molar ratio of water to surfactant) was equal to 5.5. Details of the catalysts preparation are given in our previous papers $[36,55]$. The intended loading of Pd in the catalysts was $2 \mathrm{wt} \%$.

In order to prepare $1 \mathrm{~g}$ of the catalyst with $2 \mathrm{wt} \% \mathrm{Pd}, 0.94$ $\mathrm{cm}^{3}$ of $\mathrm{PdCl}_{2}$ solution $\left(0.2 \mathrm{~mol} / \mathrm{dm}^{3}\right)$ was added to cyclohexane solution $\left(16.8 \mathrm{~cm}^{3}\right)$ of surfactant with concentration of $0.62 \mathrm{~mol} / \mathrm{dm}^{3}$. The suspension was vigorously stirred to form a transparent, clear, dark orange liquid. Palladium particles were prepared by addition of reducing agent $\left(\mathrm{NaBH}_{4}\right.$ to $\mathrm{Pd}^{2+}$ molar ratio of 10) directly to the metal salt-containing microemulsion. The reduction process occurred quickly with a change of liquid colour to black. The suspension was left for another $1 \mathrm{~h}$ under stirring. Then, the carbon support $(0.98 \mathrm{~g})$ was introduced and the system was stirred for next $1 \mathrm{~h}$. Deposition of the metal nanoparticles on the support was carried out by an addition of THF at a slow rate using an automatic syringe pump under vigorous stirring. Upon the addition of THF, the colour of liquid changed from black to grey and finally the liquid was colourless, confirming the complete deposition of metal particles on the support. The catalyst was separated by filtration, dried in air for $24 \mathrm{~h}$ and washed with a copious amount of methanol and acetone. Finally, the catalyst was washed with water till the chloride ions were removed, dried overnight in air and then at $80^{\circ} \mathrm{C}$ for $16 \mathrm{~h}$. The used washing procedure is effective to eliminate the surfactant [36].

The properties of $2 \% \mathrm{Pd} / \mathrm{VC}$ catalyst supported on commercial carbon black (Vulcan XC-72, Cabot) were reported in our previous paper dealing with its activity in the hydrogenation of cinnamaldehyde [55]. The catalyst was prepared by means of the same microemulsion method as that used in the present work.

\subsection{Physicochemical characterization}

Textural parameters were calculated from the nitrogen adsorption/desorption measurements at $196{ }^{\circ} \mathrm{C}$ using an ASAP 2020 (Micromeritics) analyser. The samples were degassed in vacuum at $350{ }^{\circ} \mathrm{C}$ for $5 \mathrm{~h}$. The specific surface areas were calculated by Brunauer-Emmett-Teller (BET) method while the pore size distributions determined by Barrett-Joyner-Halenda (BJH) model. The total pore volumes were obtained from an amount of nitrogen adsorbed at the relative pressure of 0.99 . The average pore size was calculated from the adsorption branch. 
$\mathrm{X}$-ray powder diffraction (XRD) was registered by a Bruker D2 Phase instrument with a Lynxeye detector $(\mathrm{Cu}$ $\mathrm{K} \alpha$ radiation). The average diameter of metal crystallites was calculated on the basis of the Pd (111) peak broadening according to the Scherrer equation.

The content of $\mathrm{Pd}(\mathrm{wt} \%)$ was determined by the X-ray fluorescence (XRF) method using an ARL Quant'x ED-XRF (Thermo Scientific) spectrometer with a $\mathrm{Rh}$ tube as a radiation source.

X-ray photoelectron spectroscopy (XPS) spectra were collected on a Prevac photoelectron spectrometer equipped with a hemispherical analyzer (VG Scienta R3000) using Al $\mathrm{K} \alpha(\mathrm{E}=1486.6 \mathrm{eV})$ as an $\mathrm{X}$-ray radiation source at a constant pass energy of $100 \mathrm{eV}$ for survey and high resolution modes. Powdered samples mounted on a sample holder were introduced by a load lock into an analytical chamber with a base pressure of $5 \times 10^{-9}$ mbar. The binding energy scale was calibrated using the $\mathrm{Au} 4 \mathrm{f}_{7 / 2}$ line of a cleaned gold sample at $84.0 \mathrm{eV}$. The surface composition was analyzed taking into account the areas and binding energies of $\mathrm{C} 1 \mathrm{~s}$ and $\mathrm{O} 1 \mathrm{~s}$ core levels. The spectra were fitted using CasaXPS software.

Scanning electron microscopy (SEM) images were collected by means of a JEOL JSM7500 F field emission scanning electron microscope equipped with X-ray energy dispersive (EDS) system. A secondary electron detector provided SE images, whereas a backscattered electron detector gave COMPO micrographs.

High-resolution transmission electron microscopy (HRTEM) and scanning transmission electron microscopy (STEM) studies were performed on an FEI Tecnai $\mathrm{G}^{2}$ transmission electron microscope operating at $200 \mathrm{kV}$ equipped with EDAX EDX and HAADF/STEM detectors. Samples for analysis were placed on a carbon-coated copper grid.

FT-IR spectra were recorded in the wavenumber range of $4000-650 \mathrm{~cm}^{-1}$ using a Nicolet 6700 (Thermo Scientific) spectrometer equipped with an MCT-A detector. For each sample 200 scans were taken at a resolution of $4 \mathrm{~cm}^{-1}$. Prior to the measurement, a dry powder sample was diluted with $\mathrm{KBr}$ to $0.25 \mathrm{wt} \%$ and then a $13 \mathrm{~mm}$ pellet was formed by pressing (5 tonnes).

\subsection{Catalytic tests}

The catalytic hydrogenation of furfural (Sigma-Aldrich) was studied in a batch-type glass reactor at temperature of $35^{\circ} \mathrm{C}$ and hydrogen pressure of 6 bar using 2-propanol (Avantor). The catalyst $(0.05 \mathrm{~g})$ and FU solution $\left(70 \mathrm{~cm}^{3}, 0.05 \mathrm{~mol}\right.$ $\mathrm{FU} / \mathrm{dm}^{3}$ ) were introduced into the reactor and purged with nitrogen and then with hydrogen. After heating the solution up to $35^{\circ} \mathrm{C}$, the hydrogen pressure was increased to 6 bar and the mechanical stirring was turned on (1800 rpm). Samples were taken during the reaction and analysed by GC equipped with FID detector (Perkin Elmer Clarus 500) and capillary column Elite-5 MS. The GC peaks of furan and 2-methylfuran overlapped with a broad peak of 2-propanol solvent making their analysis difficult. The reaction products furfuryl isopropyl ether (ET) and furfural diisopropyl acetal (AC) were identified by GC-MS (Thermo Electron Corporation GC equipped with TR5MS column and DSQ II Thermo Electron Corporation mass spectrometer). The carbon mass balance (CB) was calculated as the sum of the GC analysed reagents: $\mathrm{FU}+\mathrm{FA}+\mathrm{THFA}+\mathrm{ET}+\mathrm{AC}$.

\section{Results and discussion}

\subsection{Structure, texture and morphology}

The nitrogen adsorption isotherms and corresponding pore size distributions for the SBA-15 and mesostructured silica gel $\mathrm{SiO}_{2}$ applied as the templates are compared in Fig. 1a, b. The textural data collected in Table 1 shows that SBA-15 template characterizes by relatively high surface area (796 $\left.\mathrm{m}^{2} / \mathrm{g}\right)$ and total pore volume $\left(0.88 \mathrm{~cm}^{3} / \mathrm{g}\right)$ with a micropores contribution of $11.4 \%$. The high uniformity of pore sizes in the SBA-15 template is reflected by very narrow pore size distribution (Fig. 1a) with the mesopore width at the maximum of the profile at $6.5 \mathrm{~nm}$. The mesostructured silica gel template has smaller surface area $\left(296 \mathrm{~m}^{2} / \mathrm{g}\right)$ and less uniform mesopores, within range from 4 to $20 \mathrm{~nm}$ with the maximum at ca. $9.6 \mathrm{~nm}$, i.e. of wider range than in the case of SBA-15. However, the contribution of micropores (1.5\%) is very low (Table 1) which indicates a mesoporous structure of the silica template.

The SEM images presenting morphology of the carbon replicas prepared using the SBA- 15 and $\mathrm{SiO}_{2}$ templates are compared in Fig. 1. Morphology of the SBA-derived carbon $\mathrm{C}(\mathrm{ff}-\mathrm{SBA})$ consisting of the carbon nanorods is typical for the nanostructured carbons prepared with the SBA template. This morphology has been commonly discussed to reflect an inverse structure of the SBA-15 template, which is also shown in Fig. 1. Maintaining the spherical morphology characteristic of silica gel during the replication process is not a simple task. The final carbon material is usually formed as the spheres partially cracked due to their low mechanical stability. This is also the case in the disordered silica-derived $\mathrm{C}$ (ss-Si) carbon replica sample (Fig. 1b). Nevertheless, our goal in the present work was not to synthesize perfect spherical grains of replica, but rather the comparison of the Pd catalysts supported on the carbon replicas characterized by ordered (SBA-series) or disordered pore architecture $\left(\mathrm{SiO}_{2}\right.$-series).

Textural and surface properties of the synthesized carbon replicas are summarized in Tables 1 and 2. The nitrogen adsorption-desorption isotherms and pore size distributions for the SBA- and $\mathrm{SiO}_{2}$-series carbon replicas and 
Table 1 Textural properties of carbon supports and Pd-containing catalysts

\begin{tabular}{lllcl}
\hline Sample & $\begin{array}{l}\text { Surface area } \\
\left(\mathrm{m}^{2} / \mathrm{g}\right)\end{array}$ & $\begin{array}{l}\text { Pores volume } \\
\mathrm{V}_{\text {total }}\left(\mathrm{cm}^{3} / \mathrm{g}\right)\end{array}$ & $\begin{array}{l}\text { Micropores contri- } \\
\text { bution } \\
(\%)\end{array}$ & $\begin{array}{l}\text { Aver- } \\
\text { age pore } \\
\text { width } \\
(\mathrm{nm})\end{array}$ \\
\hline $\mathrm{SiO}_{2}$ (host matrix) & 296 & $0.84(0.013)^{\mathrm{c}}$ & 1.5 & 9.6 \\
$\mathrm{C}(\mathrm{ss}-\mathrm{Si})$ & 1453 & $2.85(0.12)$ & 4.2 & ca. 8 \\
$\mathrm{Pd} / \mathrm{C}(\mathrm{ss}-\mathrm{Si})$ & $1167(20 \% 15 \%)^{\mathrm{a}}$ & $2.42(0.09)$ & 3.7 & ca. 8 \\
$\mathrm{C}(\mathrm{ff}-\mathrm{Si})$ & 591 & $0.86(0.12)$ & 13.4 & ca. 8 \\
$\mathrm{Pd} / \mathrm{C}(\mathrm{ff}-\mathrm{Si})$ & $260(56 \% ; 48 \%)$ & $0.45(0.01)$ & 2.2 & ca. 8 \\
$\mathrm{SBA}-15$ (host matrix) & 796 & $0.88(0.10)$ & 11.4 & 5.3 \\
$\mathrm{C}(\mathrm{ss}-\mathrm{SBA})$ & 1273 & $1.27(0.06)$ & 4.7 & $3-4$ \\
$\mathrm{Pd} / \mathrm{C}(\mathrm{ss}-\mathrm{SBA})$ & $940(26 \% ; 49 \%)$ & $0.65(0.01)$ & 1.5 & $3-4$ \\
$\mathrm{C}(\mathrm{ff}-\mathrm{SBA})$ & 967 & $0.87(0.01)$ & 1.2 & $3-4$ \\
$\mathrm{Pd} / \mathrm{C}(\mathrm{ff}-\mathrm{SBA})$ & $748(23 \% ; 13 \%)$ & $0.76(0.06)$ & 7.9 & $3-4$ \\
$\mathrm{VC}$ & 228 & $1.77(0.05)$ & 2.8 & \\
$\mathrm{Pd} / \mathrm{VC}$ & $204(11 \% ; 20 \%)$ & $1.41(0.04)$ & 2.8 & \\
\hline
\end{tabular}

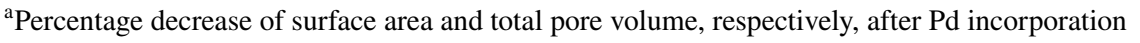

${ }^{\mathrm{b}}$ Single point total pore volume determined at $\mathrm{p} / \mathrm{p}_{0}=0.998$

${ }^{\mathrm{c}}$ Micropore volume $\mathrm{V}_{\text {micro }}$ in parentheses, $t$-plot model

${ }^{\mathrm{d}}$ Micropore contribution calculated as $\mathrm{V}_{\text {micro }} / \mathrm{V}_{\text {total }} \times 100 \%$

${ }^{\mathrm{e}} \mathrm{BJH}$ model, adsorption branch

\begin{tabular}{|c|c|c|c|c|c|c|c|}
\hline \multirow[t]{2}{*}{ Carbon material } & \multicolumn{2}{|c|}{$\begin{array}{l}\text { Surface content } \\
(\text { at } \%)\end{array}$} & \multicolumn{4}{|c|}{$\begin{array}{l}\text { Share of individual carbon species } \\
\text { (\% C 1s peak area) }\end{array}$} & \multirow[t]{2}{*}{$\mathrm{F}_{\mathrm{ox}}(\%)$} \\
\hline & $\mathrm{C}$ & $\mathrm{O}$ & $\mathrm{C}-\mathrm{OH}$ & $\mathrm{C}=\mathrm{O}$ & $\mathrm{COOH}$ & $\begin{array}{l}\pi \rightarrow \pi^{*} \\
\text { shake-up } \\
\text { satellite }\end{array}$ & \\
\hline $\mathrm{C}(\mathrm{ss}-\mathrm{Si})$ & 96.09 & 3.91 & 7.94 & 5.89 & 5.0 & 6.44 & 18.83 \\
\hline $\mathrm{C}(\mathrm{ff}-\mathrm{Si})$ & 96.40 & 3.60 & 7.03 & 6.01 & 6.19 & 4.04 & 19.23 \\
\hline $\mathrm{C}(\mathrm{ss}-\mathrm{SBA})$ & 96.22 & 3.68 & 7.27 & 4.81 & 7.51 & 2.85 & 19.59 \\
\hline C(ff-SBA) & 97.92 & 2.08 & 7.38 & 5.62 & 7.27 & 3.25 & 20.27 \\
\hline VC & 97.96 & 2.04 & 8.30 & 7.51 & 3.71 & 4.49 & 19.50 \\
\hline
\end{tabular}

Table 2 XPS characterization of carbon supports. Surface concentration of $\mathrm{C}$ and $\mathrm{O}$ (at\%) and the share (\% peak area) of the individual carbon species in $\mathrm{C}$ 1s peak. The $\mathrm{F}_{\mathrm{ox}}(\%)$ presents the sum of peak areas of all oxidized carbon groups in the C 1s peak corresponding Pd-containing catalysts are presented in Figs. 2 and 3, respectively.

The shapes of the isotherms for all the SBA-series samples, before and after Pd intercalation, are of type IV with a $\mathrm{H} 1$ hysteresis loop observed at $\mathrm{p} / \mathrm{p}_{\mathrm{o}}$ between 0.3 and 0.8 (Fig. 2). A steep step in the adsorption isotherm observed for both carbon replicas and Pd-containing samples reveals the ordered pore structures with mesopores of narrow size distribution evidenced by the pore distribution profiles (Fig. 2). In both SBA-derived carbon replicas the mesopores of size within the range of $2-5 \mathrm{~nm}$ are seen with an average pore width of approximately $3.5 \mathrm{~nm}$. However, the mesopores are of somewhat narrower range in the furfuryl alcohol-derived carbon sample than in the sucrose-arising carbon (Fig. 2a).

Both carbon replicas, C(ss-SBA) and C(ff-SBA) possess a higher surface area $\left(1273\right.$ and $\left.967 \mathrm{~m}^{2} / \mathrm{g}\right)$ compared to that of SBA-15 template $\left(796 \mathrm{~m}^{2} / \mathrm{g}\right)$, especially the sucrose-derived sample (Table 1). The total porosity of the sucrose-derived carbon $\left(1.27 \mathrm{~cm}^{3} / \mathrm{g}\right)$ exceeds distinctly the total porosity of furfuryl alcohol-derived sample $\left(0.87 \mathrm{~cm}^{3} / \mathrm{g}\right)$, being comparable to that of SBA- 15 template $\left(0.88 \mathrm{~cm}^{3} / \mathrm{g}\right)$. The sucrosederived $\mathrm{C}(\mathrm{ss}-\mathrm{SBA})$ carbon characterizes also by slightly higher microporosity (4.7\%) than that of the furfuryl alcohol-derived C(ff-SBA) sample (1.2\%).

The low-angle X-ray diffraction patterns of the SBA-15 series samples exhibit the reflections below $2 \theta=5^{\circ}$ which is typical for the highly ordered mesostructure. They are characteristic of the hexagonal arrangement of the mesochannels with the space group of P6mm (Fig. 2c). However, the diffraction lines indexed as (100), (110) and (200) in the $\mathrm{C}$ (ff-SBA) carbon replica prepared with furfuryl alcohol are located at somewhat lower $2 \theta$ values $(1.12,1.90$ and $2.20^{\circ}$ respectively) compared to the C(ss-SBA) sample synthesized with the sucrose precursor $\left(1.13,1.93\right.$ and $\left.2.24^{\circ}\right)$ 
Fig. 1 Nitrogen adsorption isotherms and pore size distributions (inset) of SBA-15 (a) and mesoporous silica gel $\mathrm{SiO}_{2}(\mathbf{b})$ templates and the morphology of both silica templates and corresponding carbon replicas $\mathrm{C}$ (ffSBA) and C(ss-Si) observed by SEM in SEI mode
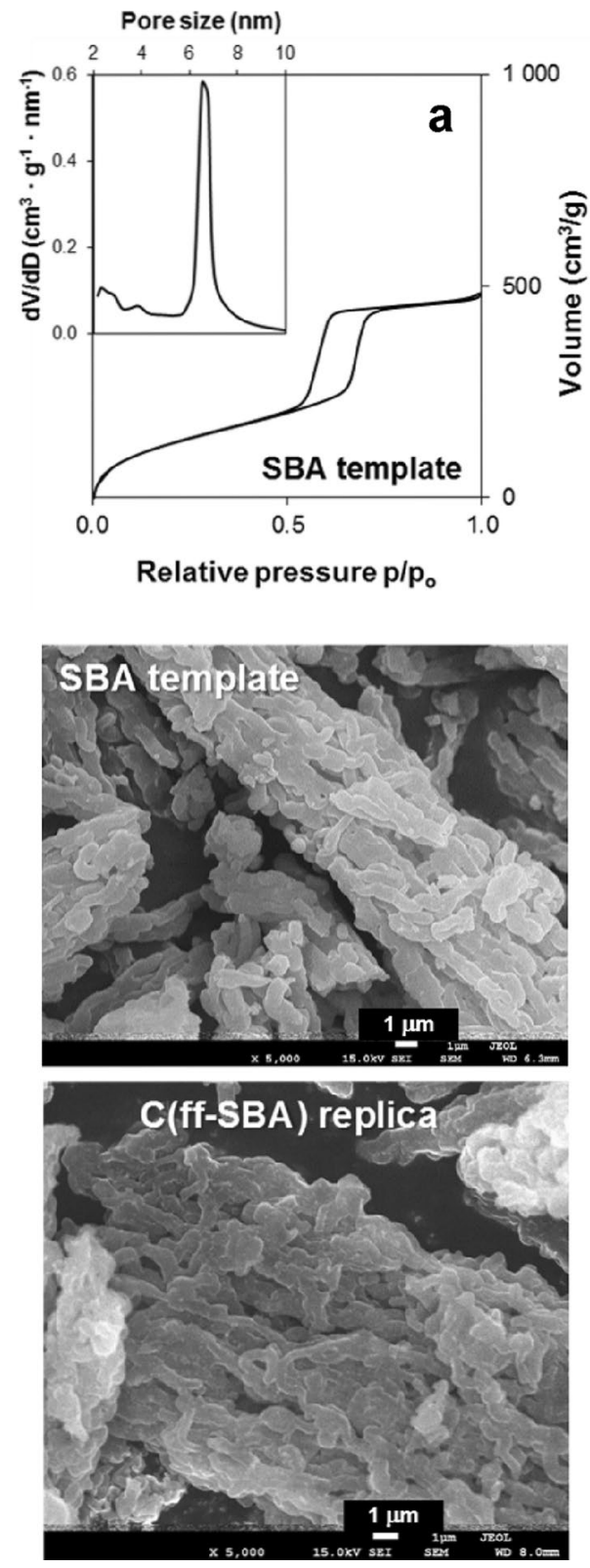
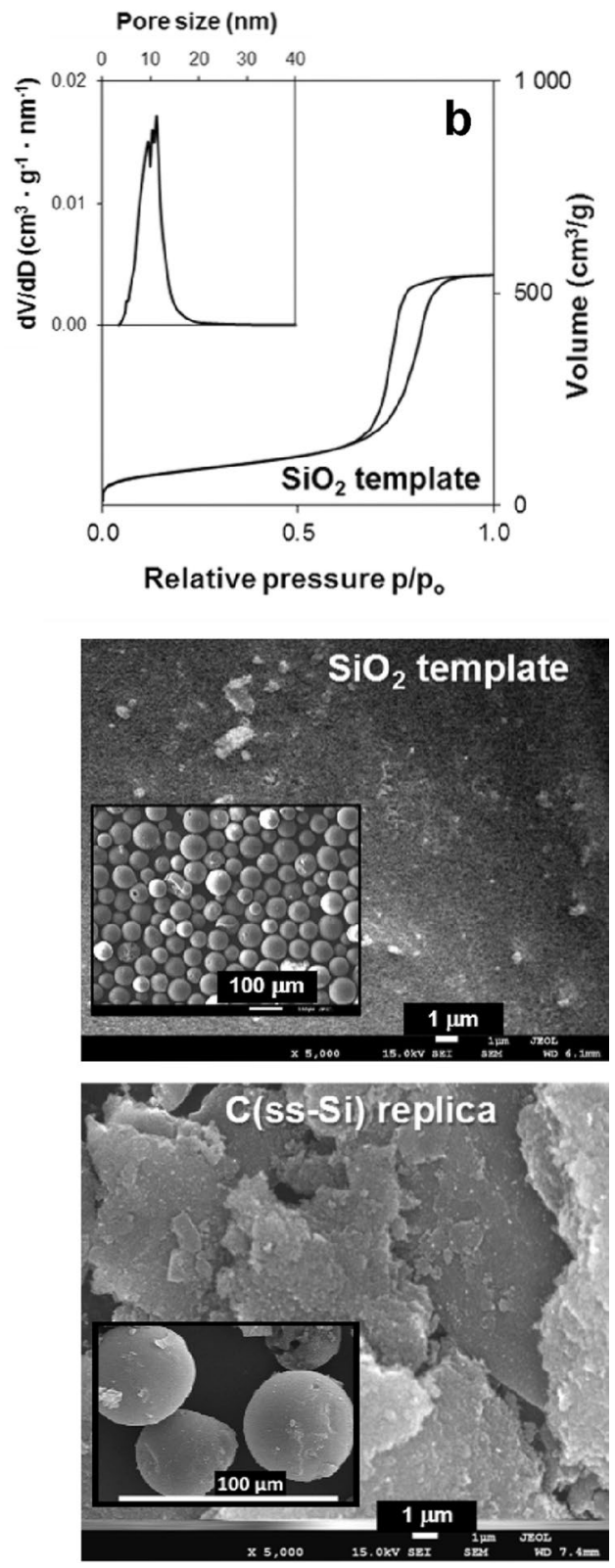

(Fig. 2c). The lattice parameter $a=9.03 \mathrm{~nm}$ for the sucrose arising carbon $\mathrm{C}$ (ss-SBA) is therefore slightly lower than $a=9.11 \mathrm{~nm}$ calculated for the furfuryl alcohol derived carbon C(ff-SBA). Comparing these values, differences in the average pore size of both replicas $(3.95 \mathrm{~nm}$ for C(ss-SBA) and $3.58 \mathrm{~nm}$ for C(ff-SBA)) should be kept in mind. Since the $a$ parameter corresponds to the distance between the centres of adjacent pores, the wall thickness of the furfuryl alcohol-derived C(ff-SBA) carbon replica is determined to be $5.53 \mathrm{~nm}$, being significantly higher than $5.08 \mathrm{~nm}$ in the sucrose-derived $\mathrm{C}(\mathrm{ss}-\mathrm{SBA})$ carbon. It can be seen that at the cost of the thinner pore walls in the sucrose-derived sample, it tends to have a larger available porous surface $\left(1273 \mathrm{~cm}^{3} / \mathrm{g}\right.$ ), which is one of the feature characteristic of the carbons synthesized with sucrose precursor.

The wide-angle XRD patterns show the weak and broad peaks around $2 \theta=20^{\circ}$ and $44^{\circ}$ which correspond to the (002) and (101) reflections arising from the graphitic domains (Fig. 4a). The low intensity of these peaks indicates mostly amorphous structure and low graphitic order. This result is consistent with the previous observation that nongraphitizable sources, such as sucrose and furfuryl alcohol, produced carbon replicas of low crystallinity [56].

The intended palladium loading in the prepared catalysts was $2 \mathrm{wt} \%$. However, this loading is obtained in the Pd/VC catalyst only, whereas the carbon replicas-supported catalysts have lower Pd content 1.1-1.4 wt\% (Table 3). 
Fig. 2 Nitrogen adsorption isotherms and pore size distributions for SBA-derived carbon replicas (a) and Pd-supported catalysts (b) together with XRD patterns (c)
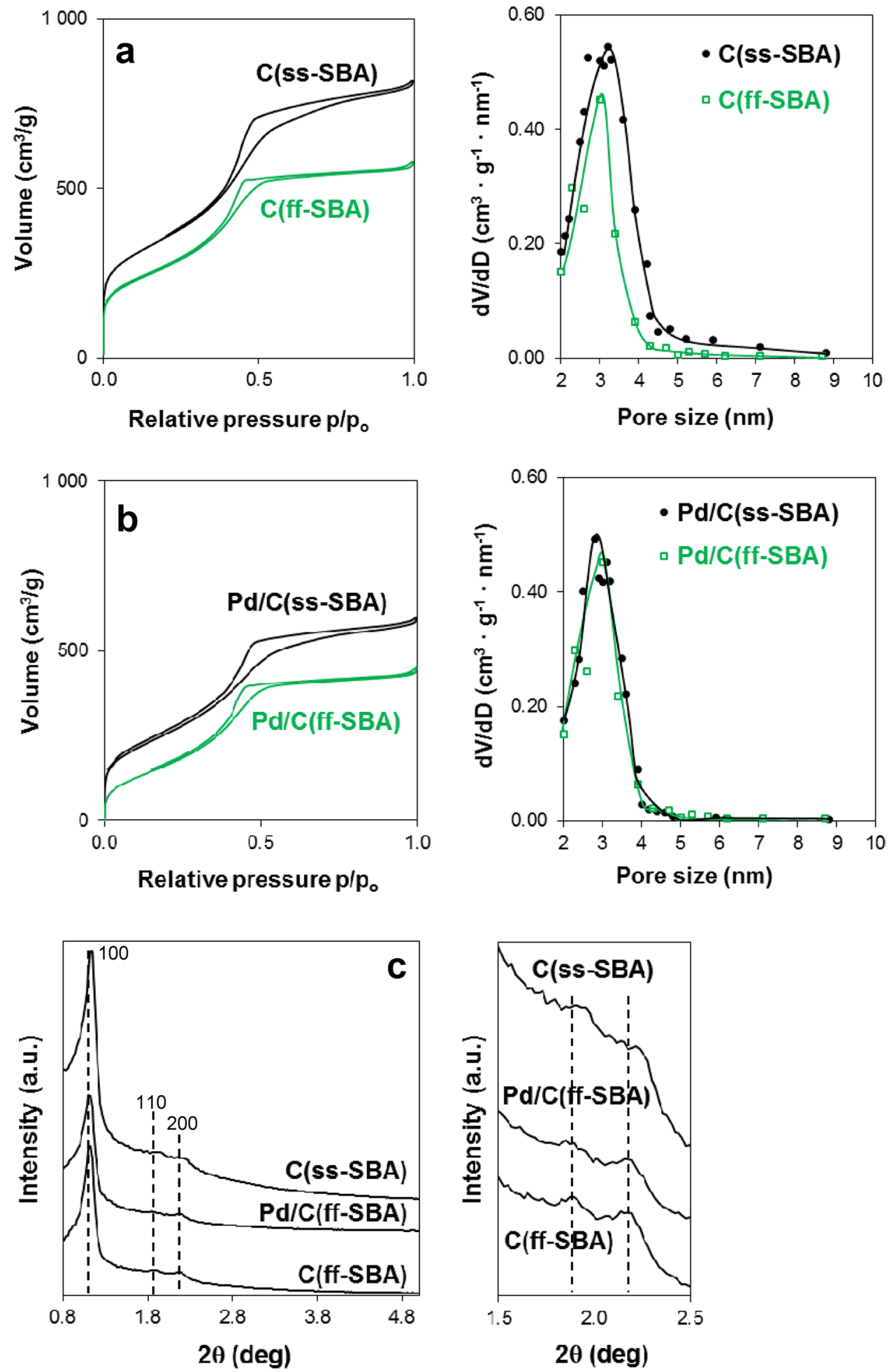

After palladium incorporation the specific surface areas of both C(ss-SBA) and C(ff-SBA) carbons are reduced by ca. 23-26\%, however, the ordered mesopore structures are preserved as evidenced by the nitrogen adsorption isotherms and the pore size distribution profiles (Fig. 2b). These profiles clearly indicate that changes in the porosity are more pronounced in the sucrose-derived sample which lost ca. $49 \%$ of total pore volume (from 1.27 to $0.65 \mathrm{~cm}^{3} / \mathrm{g}$; Table 1) assisted by a decrease of the micropores contribution to ca. $1.5 \%$. On the other hand, the comparison of the pore distribution profiles for the furfuryl alcohol-derived carbons before and after Pd insertion indicates only slight decrease 
of the mesoporosity (Fig. 2b), whereas the microporosity grows from 1.2 to $7.9 \%$ (Table 1 ).

The nitrogen adsorption isotherms of carbon replicas prepared with the nanostructured $\mathrm{SiO}_{2}$ template are of type IV and exhibit hysteresis loops characteristic of mesoporous structure (Fig. 3a). However, in contrast to the SBA-derived ordered carbon structures, the samples prepared with the nanostructured $\mathrm{SiO}_{2}$ template display different textural characteristics, depending on the type of carbon precursor used. The sucrose-derived $\mathrm{C}(\mathrm{ss}-\mathrm{Si})$ carbon characterizes by very high surface area $\left(1453 \mathrm{~m}^{2} / \mathrm{g}\right)$ and total porosity $\left(2.85 \mathrm{~g} / \mathrm{cm}^{3}\right)$ which are much higher compared to the furfuryl alcohol-derived C(ff-Si) carbon $\left(591 \mathrm{~cm}^{2} / \mathrm{g}\right.$ and 0.86 $\mathrm{cm}^{3} / \mathrm{g}$ ) (Table 1). The latter $\mathrm{C}(\mathrm{ff}-\mathrm{Si}$ ) carbon sample characterizes also by relatively high microporosity of $13.4 \%$ which much exceeding the microporosity of only $4.2 \%$ in the sucrose-derived carbon. The pore distribution profiles show mesopores in relatively broad range mainly $2-15 \mathrm{~nm}$ with an average width of $8 \mathrm{~nm}$ (Fig. 3), which is below that of the $\mathrm{SiO}_{2}$ template and is consistent with other authors [24].

The small and broad reflections at $2 \theta=20^{\circ}$ and $44^{\circ}$ characteristic of graphitic structure observed in the XRD pattern of both $\mathrm{SiO}_{2}$-templated carbon samples (Fig. 4a) indicate low crystalline carbons similarly to the SBA-templated counterparts. In the XRD patterns of the Pd-containing carbons the distinct reflection at $2 \theta \sim 40^{\circ}$ indexed to the (111) plane of face centred cubic (fcc) crystalline Pd occurs (Fig. 4b). The average Pd crystallite size (estimated by Scherrer equation from the $\operatorname{Pd}(111)$ line broadening) is $4-5 \mathrm{~nm}$ in all studied catalysts. The obtained metal particle size agrees well with our previous results dealing with the palladium and other monometallic ( $\mathrm{Ru}, \mathrm{Pt}, \mathrm{Ir}, \mathrm{Au})$ and Pd-based bimetallic catalysts synthesized using the same microemulsion composition [57]. It should be noticed, however, that whatever the type of silica template, the reflection at approximately $2 \theta=20^{\circ}$ is narrower and more intense and locates at a slightly higher $2 \theta$ value in the patterns of furfuryl alcohol-derived samples in comparison to the sucrose-derived counterparts (Fig. 4a). It reveals somewhat higher graphitization degree of the furfuryl alcohol-derived carbons than that of the sucrose-derived samples, consistent with previous observations [56]. This implies that the carbon materials obtained from furfuryl alcohol precursor characterize by somewhat higher
Fig. 3 Nitrogen adsorption isotherms and pore size distributions for $\mathrm{SiO}_{2}$-derived carbon replicas (a) and Pd-supported catalysts (b)
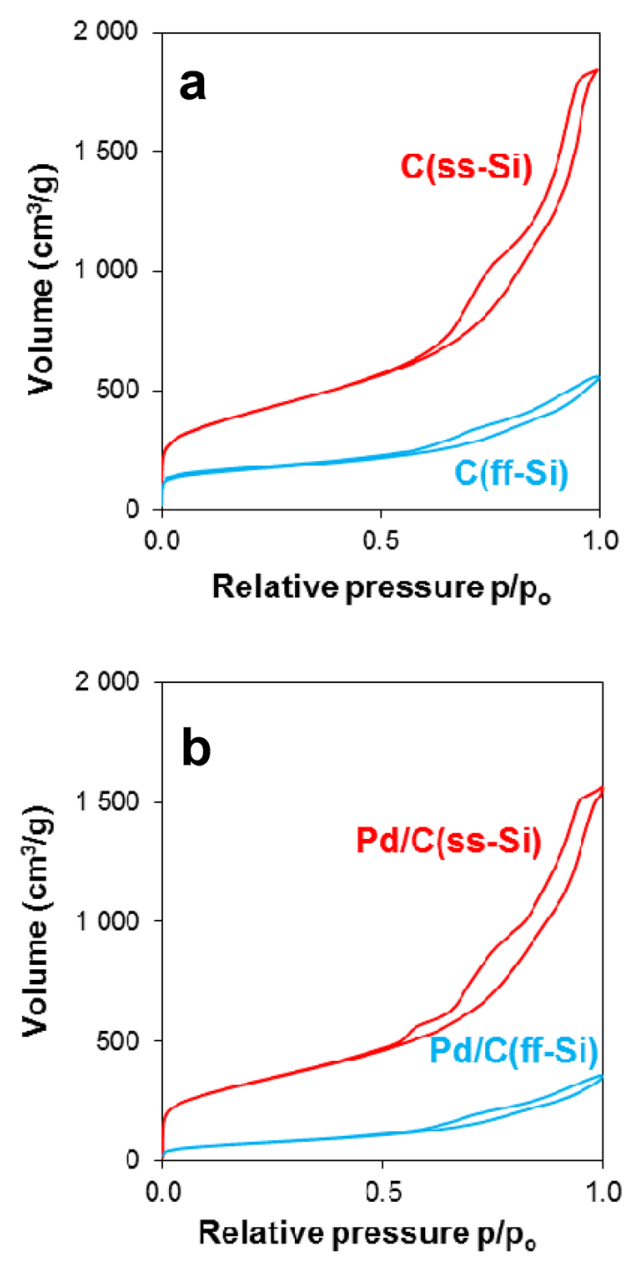
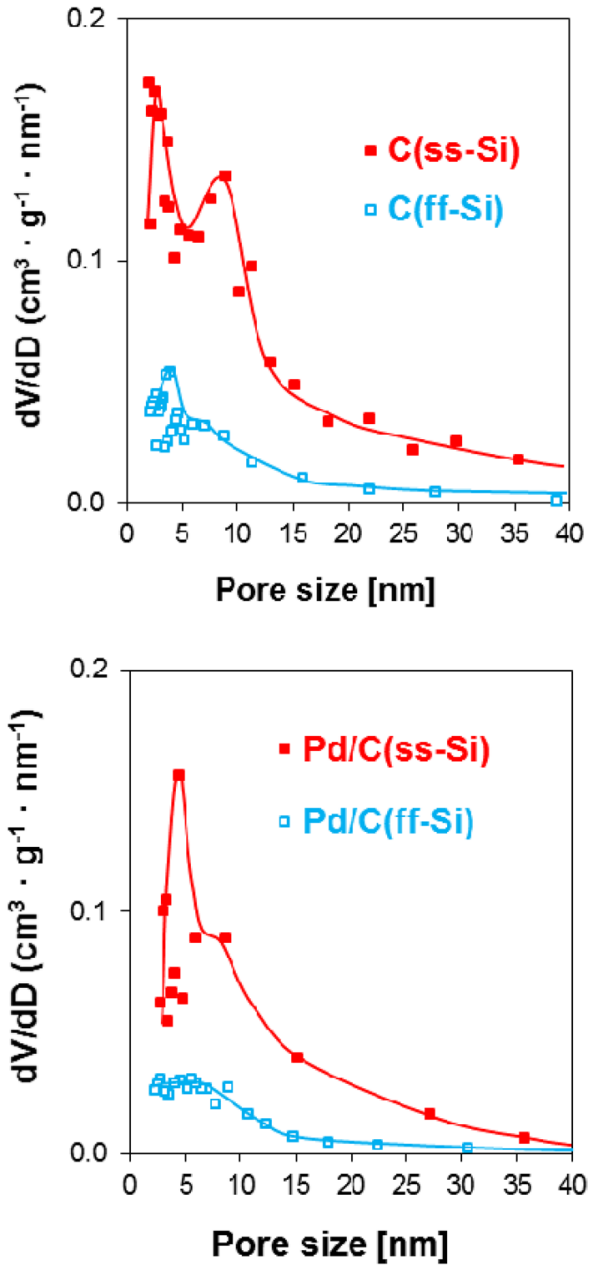
Fig. 4 The wide-angle XRD patterns for carbon replicas (a) and catalysts (b)

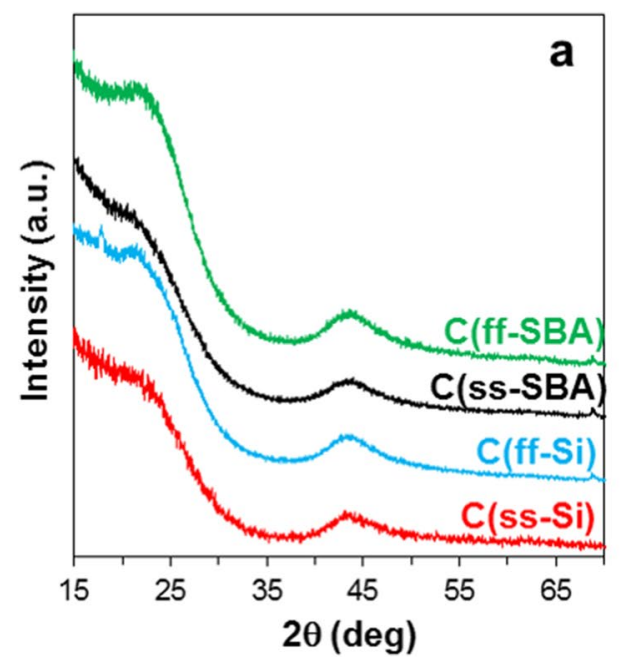

polyaromatic character and higher ordering of graphene layers comparing to their counterparts synthesized with sucrose precursor.

The incorporation of $\mathrm{Pd}(1.3 \mathrm{wt} \%)$ to the $\mathrm{C}(\mathrm{ss}-\mathrm{Si})$ carbon sample reduces surface area by $20 \%$ only (from 1453 to $1167 \mathrm{~m}^{2} / \mathrm{g}$ ) accompanied by a small reduction of total porosity, by $15 \%$ only. Minor changes in the pore distribution also occur but a low microporosity is almost preserved. The incorporation of similar Pd loading, $1.07 \mathrm{wt} \%$ in the furfuryl alcohol-derived $\mathrm{C}(\mathrm{ff}-\mathrm{Si})$ carbon replica results in much more pronounced changes in textural parameters. The BET surface area and total porosity decrease by half, $56 \%$ and $48 \%$, respectively. The observed large loss of surface area could be ascribed to a significant decrease of microporosity from 13.4 to $2.2 \%$ (Table 1 ).

The carbon black Vulcan XC-72 (VC) is the most commonly used support material for the Pt, Pt, Rucontaining electrocatalysts developed for fuel cells. The textural features and morphology of $\mathrm{VC}$ carbon and synthesized $2 \% \mathrm{Pd} /$ VC catalyst are collected in Fig. 5. The VC carbon characterizes by surface area of $228 \mathrm{~m}^{2} / \mathrm{g}$ and much higher contribution of mesopores $\left(1.72 \mathrm{~cm}^{3} / \mathrm{g}\right)$ than micropores $(0.048$ $\mathrm{cm}^{3} / \mathrm{g}$ ). The pore size distribution (Fig. 5b) indicates the presence of pores within relatively wide range, mainly 2-6 $\mathrm{nm}$ with a large contribution of pores having a diameter below $3 \mathrm{~nm}$ (small mesopores). The XRD pattern of the VC sample presenting two broad reflections at $2 \theta=24.7^{\circ}$ and $43.7^{\circ}$ is consistent with previous reports showing high contribution of graphitic domains in this material [58, 59]. Electron microscopic images of VC carbon (Fig. 5d,e) show the morphology consisting of aggregated quasi-spherical particles with a diameter of 30-50 nm (Fig. 5e). HRTEM micrograph in Fig. 5d demonstrates that the spherical carbon particles are constituted of groups of graphene planes $[60,61]$. After Pd intercalation, the specific surface area and total porosity are reduced to some extent (by $11 \%$ and $20 \%$, respectively) accompanied by a 50\% decrease of the volume associated to the mesopores as the volume of micropores is weakly reduced (Table 1).

\subsection{Surface composition}

It is well known that various oxygenated functionalities, including phenolic, ether, carbonyl, carboxylic, are typically distributed on the surface of carbon samples. The FT-IR and XPS techniques are usually applied to examine these oxygenated carbon functionalities. The FT-IR spectra of our carbon samples demonstrate several weak bands within the $1000-1800 \mathrm{~cm}^{-1}$ region arising from the oxygen-containing carbon groups (Fig. S1a). There is no essential difference in the spectra between sucrose- and furfuryl alcohol-derived carbon samples. The peaks at 1000-1100, 1300-1400 and $1636 \mathrm{~cm}^{-1}$ correspond to $\mathrm{C}-\mathrm{O}-\mathrm{C}, \mathrm{C}-\mathrm{OH}$ and $\mathrm{C}=\mathrm{C}$ vibration frequencies, respectively. The bands below $960 \mathrm{~cm}^{1}$ are attributed to the out-of-plane bending vibrations of the $\mathrm{C}-\mathrm{H}$ bonds. These bands are not observable in the spectrum of VC carbon because of high contribution of graphitic structures which manifests as the distinct band at $1630 \mathrm{~cm}^{-1}$ corresponding to the $\mathrm{C}=\mathrm{C}$ vibrations, similarly to previous data $[58,62]$. However, the band at $1630 \mathrm{~cm}^{-1}$ can be also related to the vibrations of quinone groups and/or adsorbed water.

The XPS analysis indicates that the surface of all carbon replicas is dominated by the carbon and its concentration ranged from 96 to 98 at\% (Table 2), whereas the oxygen content is very low ca. 2-4 at $\%$. Thus, the carbonization process in inert atmosphere significantly decreases the oxygen quantity. These results are consistent with previous data showing that the low oxygen content on the surface of carbon replicas is an indication of well-ordered surface (without or with only very small concentration of defects) [63].

The $\mathrm{C}$ 1s spectra of all carbon replicas are dominated by an intense and asymmetrical graphite peak at $\mathrm{BE}=284.4 \mathrm{eV}$ 
assisted by a small high-energy peak at binding energy above $290.4 \mathrm{eV}$ corresponding to $\pi \rightarrow \pi^{*}$ plasmon excitation [63, 64]. The observed $C 1$ s spectra are typical for carbonaceous samples with a graphite-like, polyaromatic surface consisting mainly of graphene layers. In the XPS spectra of all carbon replicas the $\mathrm{C} 1 \mathrm{~s}$ peaks components corresponding to the oxygenated carbon groups $\mathrm{C}-\mathrm{OH}(286.3 \mathrm{eV}), \mathrm{C}=\mathrm{O}$ $(287.4 \mathrm{eV})$ and $\mathrm{COOH}(288.9 \mathrm{eV})$ are of considerably low intensity (Fig. S2).

The percentage areas of individual carbon groups in $\mathrm{C}$ 1s peak are collected in Table 2. It can be seen that all the $\mathrm{C}-\mathrm{OH}, \mathrm{C}=\mathrm{O}$ and $\mathrm{COOH}$ groups appear in the carbon materials and there is no essential difference between the contribution of particular oxygenated groups.

The peaks fitted to the $\mathrm{O} 1 \mathrm{~s}$ region of studied carbon samples provided information complementary to that from the $\mathrm{C} 1 \mathrm{~s}$ spectra (Fig. S3). The $\mathrm{O} 1 \mathrm{~s}$ regions also display some asymmetric tailing. The peak components at energy of $531.1 \mathrm{eV}(\mathrm{C}=\mathrm{O}$ in quinone), $532.9 \mathrm{eV}$ (oxygen in $\mathrm{OH}$, ether, ester, anhydride and carboxyl), $534.5 \mathrm{eV}$ (ether oxygen in ester, anhydride and $\mathrm{OH}$ in carboxyl group) and $536.9 \mathrm{eV}$ (adsorbed water) are observed.

All the Pd-containing carbon samples were also studied by XPS (Fig. S4) and FT-IR techniques (Fig. S1). The FT-IR spectra of Pd-containing catalysts (Fig. S1b) present the same set of the bands of oxygenated carbon groups as those in the spectra of corresponding Pd-free carbon samples, before the Pd intercalation. However, in the spectra of Pdcontaining catalysts some of bands, especially the ones characteristic of $\mathrm{OH}$ groups seem to be wider (ca. $1100 \mathrm{~cm}^{-1}$ ) or more intense (at $1600-1630 \mathrm{~cm}^{-1}$ ). This effect manifests weakly, nevertheless, it is more distinctly observable in the spectra of $\mathrm{Pd} / \mathrm{C}(\mathrm{ff}-\mathrm{Si}$ ) and $\mathrm{Pd} / \mathrm{C}(\mathrm{ff}-\mathrm{SBA})$ catalysts, both supported on furfuryl alcohol-derived carbons (Fig. S1b).

The XPS spectra of catalysts show the presence of carbon $(\mathrm{C} 1 \mathrm{~s})$, oxygen $(\mathrm{O} 1 \mathrm{~s})$, and palladium ( $\mathrm{Pd} 3 \mathrm{~d})$ elements, at atomic composition (at\%) summarized in Table 3. The XPS data obtained for the $\mathrm{Pd} / \mathrm{VC}$ catalysts are also provided. Similarly as in the case of carbon replicas, the $\mathrm{C} 1 \mathrm{~s}$ energy region consists of an intense and dominating graphite peak located at $\mathrm{BE}=284.4 \mathrm{eV}$ and small peak components related to the oxygenated carbon groups $(\mathrm{C}=\mathrm{O}, \mathrm{C}-\mathrm{OH}$ and $\mathrm{COOH})$ as well as the small peak at energy above $290.4 \mathrm{eV}$ corresponding to $\pi \rightarrow \pi^{*}$ plasmon excitation (Fig. S4). The total percentage fraction (\%) of the oxygenated groups in the $\mathrm{C} 1 \mathrm{~s}$ peak is somewhat higher in $\mathrm{Pd} / \mathrm{C}(\mathrm{ss}-\mathrm{Si})$ and $\mathrm{Pd} / \mathrm{C}(\mathrm{ss}-\mathrm{SBA})$ catalysts with sucrose-derived carbons (19.69 and 24.03\%, respectively) relative to the counterparts with furfuryl alcohol-derived carbons (15.0 and $19.89 \%$, Table 4). Furthermore, there is no essential difference in the percentage area of the peaks related to particular oxygenated carbon groups (Table 4) between the catalysts. Nevertheless, it is observed that the $\mathrm{C}-\mathrm{OH}$ groups dominates in all the catalysts, except the $\mathrm{Pd} / \mathrm{C}$ (ff-SBA) catalyst with the dominating $\mathrm{COOH}$ ones.

The $\mathrm{C} 1 \mathrm{~s}$ spectra of $\mathrm{VC}$ carbon (Fig. S2) and Pd/VC catalyst (Fig. S4) show the main component (45\% of peak area) at energy around $284.4 \mathrm{eV}$ consistent with the literature reports attributing this binding energy to the graphitic carbon [62]. A subpeak at ca. $285 \mathrm{eV}$ (28\% of peak area) is due to hydrocarbons on the surface of grains coming mainly from the contamination of the powder surface. Similarly as in the carbon replicas-containing samples, the sets of peaks within higher binding energies correspond to oxygenated carbon groups, whereas the high-energy peak component centred at $291.2 \mathrm{eV}$ (ca. 3-6\%) corresponds to $\pi \rightarrow \pi^{*}$ plasmon excitation [58, 65] (Table 4).

The XPS Pd 3d core level spectra of catalysts (Fig. S5) and the corresponding binding energies of $\mathrm{Pd} 3 \mathrm{~d}_{5 / 2}$ peak are summarized in Table 3. It should be pointed out that no chlorine residuals from the metal precursors were found in the XPS survey spectra. The XPS analysis provided data including surface concentration of palladium $\left(\mathrm{Pd}_{\mathrm{S}}\right)$
Table 3 Characterization of catalysts by XPS technique; surface content of $\mathrm{C}, \mathrm{O}$ and $\mathrm{Pd}$ (at\%) and surface atomic ratio of oxygen to carbon and oxygen to palladium

\begin{tabular}{|c|c|c|c|c|c|c|c|c|}
\hline \multirow[t]{2}{*}{ Catalyst } & \multirow[t]{2}{*}{$\begin{array}{l}\text { Pd bulk } \\
(\mathrm{wt} \%)\end{array}$} & \multicolumn{4}{|c|}{$\begin{array}{l}\text { XPS data, surface content } \\
\text { (at } \%)\end{array}$} & \multicolumn{2}{|c|}{ Surface atomic ratio } & \multirow[t]{2}{*}{$\begin{array}{l}\mathrm{Pd} 3 \mathrm{~d}_{5 / 2} \\
\mathrm{BE} \mathrm{eV}\end{array}$} \\
\hline & & $\overline{\mathrm{C}}$ & $\mathrm{O}$ & $\mathrm{Pd}_{\mathrm{S}}$ & $\overline{\mathrm{O} / \mathrm{C}}$ & $\overline{\mathrm{O}_{\mathrm{S}} / \mathrm{Pd}_{\mathrm{S}}}$ & $\mathrm{Pd}_{\mathrm{S}} / \mathrm{Pd}_{\mathrm{B}}$ & \\
\hline $\mathrm{Pd} / \mathrm{C}(\mathrm{ss}-\mathrm{Si})$ & 1.30 & 88.6 & 9.8 & 1.47 & 0.11 & 6.7 & 10.7 & $\begin{array}{l}335.5(0.89)^{\mathrm{b}} \\
337.2(0.11)\end{array}$ \\
\hline $\mathrm{Pd} / \mathrm{C}(\mathrm{ff}-\mathrm{Si})$ & 1.07 & 87.5 & 11 & 1.09 & 0.12 & 10.1 & 10.0 & $\begin{array}{l}335.5(0.87) \\
337.2(0.13)\end{array}$ \\
\hline $\mathrm{Pd} / \mathrm{C}(\mathrm{ss}-\mathrm{SBA})$ & 1.12 & 88.6 & 9.5 & 1.85 & 0.11 & 5.13 & 16.1 & $\begin{array}{l}335.6(0.91) \\
337.3(0.09)\end{array}$ \\
\hline $\mathrm{Pd} / \mathrm{C}(\mathrm{ff}-\mathrm{SBA})$ & 1.40 & 88.9 & 8.7 & 2.45 & 0.10 & 3.55 & 16.8 & $\begin{array}{l}335.4(0.86) \\
337.1(0.14)\end{array}$ \\
\hline $\mathrm{Pd} / \mathrm{VC}$ & 2.0 & 95.5 & 3.8 & 0.28 & 0.04 & 13.64 & 1.3 & $\begin{array}{l}335.6(0.61) \\
336.5(0.39)\end{array}$ \\
\hline
\end{tabular}

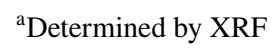

${ }^{\mathrm{b}}$ Contribution to total $\mathrm{Pd} 3 \mathrm{~d}_{5 / 2}$ peak area 
Fig. 5 Nitrogen adsorption isotherms (a), pore size distributions (b), XRD patterns (c), HRTEM image (d) and SEM images (e) for Vulcan XC-72 carbon (VC) and $\mathrm{Pd} / \mathrm{VC}$ catalyst
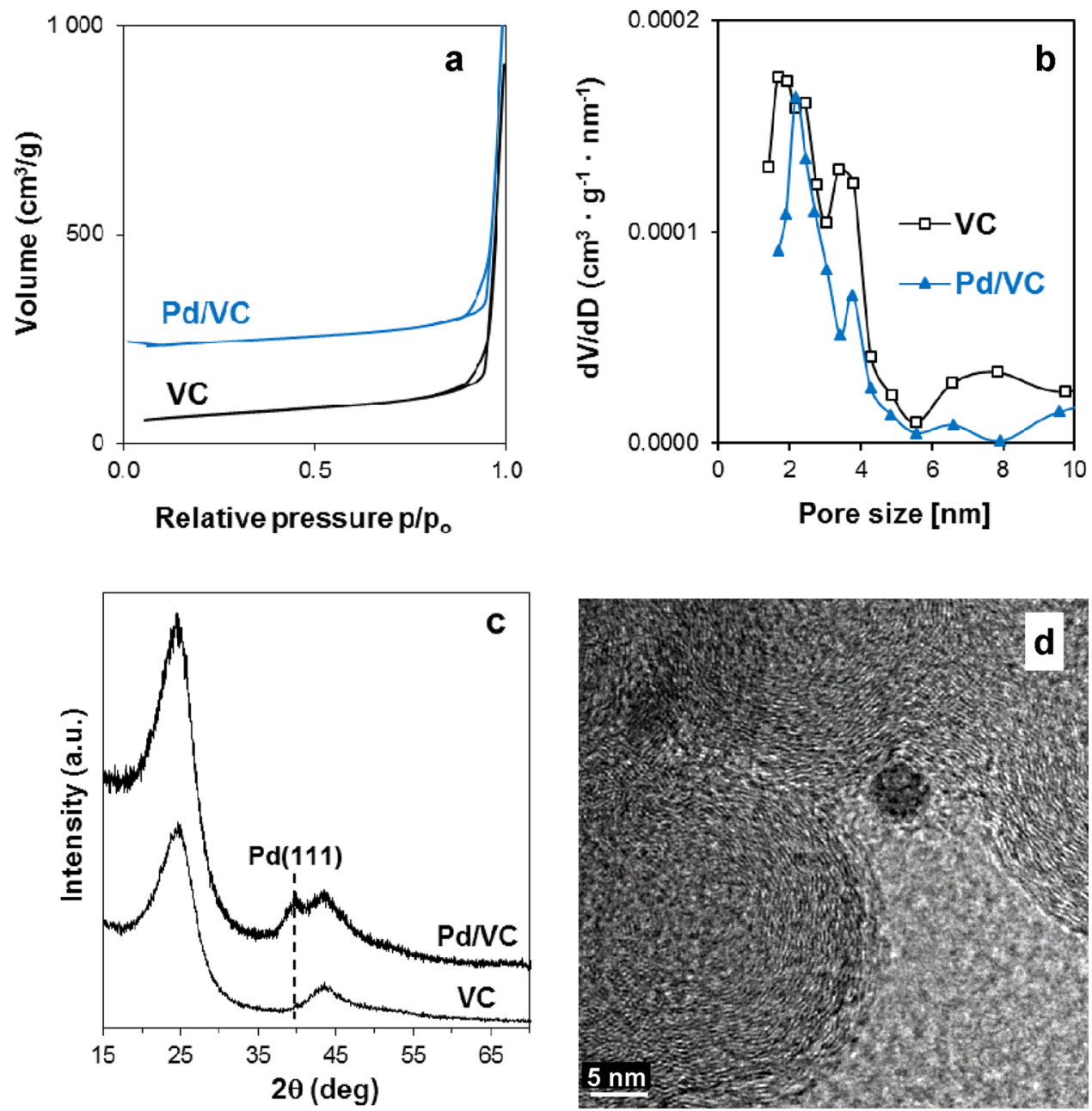

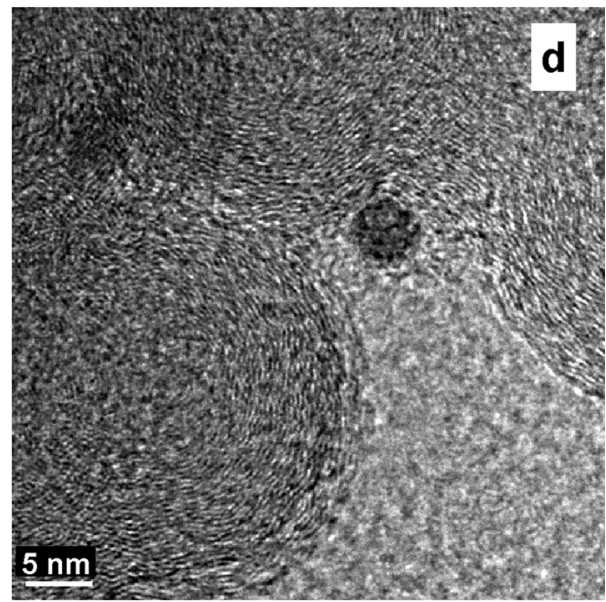

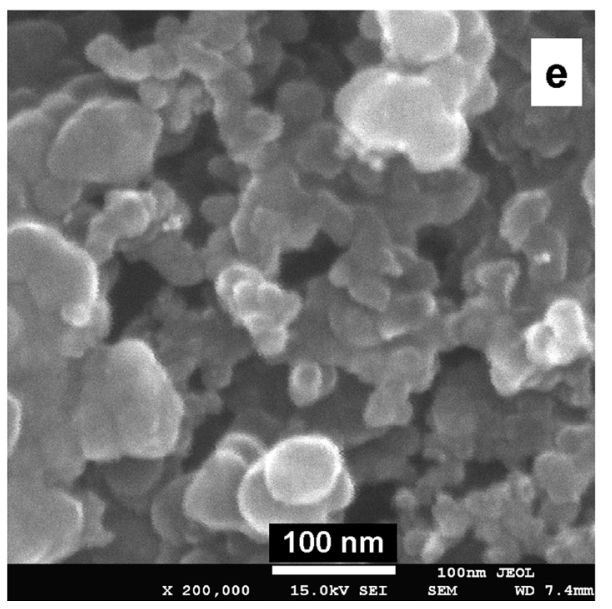

Table 4 Characterization of catalysts by XPS technique; the percentage area of the individual carbon species in $\mathrm{C}$ 1s peak. The $\mathrm{F}_{\mathrm{ox}}(\%)$ presents the sum of percentage areas of all oxidized carbon groups in $\mathrm{C}$ 1s peak

\begin{tabular}{lcccccc}
\hline Catalyst & $\mathrm{C}-\mathrm{C}$ & $\mathrm{C}-\mathrm{OH}$ & $\mathrm{C}=\mathrm{O}$ & $\mathrm{COOH}$ & $\pi-\pi^{*}$ & $\mathrm{~F}_{\text {ox }}(\%)$ \\
\hline $\mathrm{Pd} / \mathrm{C}(\mathrm{ss}-\mathrm{Si})$ & 75.42 & 7.57 & 7.03 & 5.09 & 4.92 & 19.69 \\
$\mathrm{Pd} / \mathrm{C}(\mathrm{ff}-\mathrm{Si})$ & 80.57 & 7.40 & 4.24 & 3.36 & 3.63 & 15.00 \\
$\mathrm{Pd} / \mathrm{C}(\mathrm{ss}-\mathrm{SBA})$ & 70.54 & 9.78 & 7.88 & 6.37 & 5.43 & 24.03 \\
$\mathrm{Pd} / \mathrm{C}(\mathrm{ff}-\mathrm{SBA})$ & 77.38 & 6.57 & 6.21 & 7.11 & 2.73 & 19.89 \\
$\mathrm{Pd} / \mathrm{VC}$ & 56.38 & 18.51 & 11.6 & 6.31 & 6.09 & 36.44 \\
\hline
\end{tabular}


and the electronic features of the Pdspecies (Table 3). The obtained Pd data correspond to the percentage of palladium atoms in surface layers, as the $\mathrm{X}$-ray penetration does not exceed ca. 6-8 nm. Based on the obtained results, the depth of palladium penetration leading to an enrichment of the carbon grains surface in Pd is evaluated, similarly to Calvo et al. [66] who studied an effect of catalyst surface composition on the activity and selectivity of activated carbons-supported Pd catalysts in hydrodechlorination reactions.

As described earlier, the carbon replicas-supported catalysts have similar Pd bulk content 1.1-1.4 wt\% (Table 3). However, they differ distinctly in the XPS-derived Pd surface concentration, which is the highest in both catalysts supported on the SBA-15-derived carbons (1.85 and 2.45 at\% Pd). Somewhat lower, but still high Pd surface concentration of 1.47 at $\%$ is found in the $\mathrm{Pd} / \mathrm{C}(\mathrm{ss}-\mathrm{Si})$ catalyst. The $\mathrm{Pd} / \mathrm{C}(\mathrm{ff}-\mathrm{Si})$ and $\mathrm{Pd} / \mathrm{VC}$ catalysts exhibit lower Pd surface concentration, which is especially low in the $\mathrm{Pd} / \mathrm{VC}$ catalyst $(0.28$ at\% $\mathrm{Pd})$. The palladium surface to bulk atomic ratio $\mathrm{Pd}_{\mathrm{S}} / \mathrm{Pd}_{\mathrm{B}}$ (where $\mathrm{Pd}_{\mathrm{S}}$ is determined by XPS, and $\mathrm{Pd}_{\mathrm{B}}$ by $\mathrm{XRF}$ ) could provide rough information about an enrichment of the carbon surface in Pd when compared to the bulk. The calculated results (Table 3 ) reveal a distinct Pd enrichment of outer surface in both SBA-series catalysts. The $\mathrm{Pd}_{\mathrm{S}} / \mathrm{Pd}_{\mathrm{B}}$ ratios are as high as 16.1 and 16.8 for the $\mathrm{Pd} / \mathrm{C}$ (ss-SBA) and $\mathrm{Pd} / \mathrm{C}$ (ff-SBA) catalysts, respectively. In contrast, the atomic ratio of $\mathrm{Pd}_{\mathrm{S}} / \mathrm{Pd}_{\mathrm{B}}=1.3$ in the $\mathrm{Pd} / \mathrm{VC}$ catalyst indicates easy penetration of $\mathrm{Pd}$ inside the grains of $\mathrm{VC}$ carbon. The penetration of $\mathrm{Pd}$ inside the grains of $\mathrm{SiO}_{2}$-derived carbon replicas also occur, but to distinctly lower extent as the $\mathrm{Pd}_{S} / \mathrm{Pd}_{\mathrm{B}}$ atomic ratios are high, 10.7 and 10.0 for the $\mathrm{Pd} / \mathrm{C}(\mathrm{ss}-\mathrm{Si})$ and $\mathrm{Pd} / \mathrm{C}(\mathrm{ff}-\mathrm{Si})$ catalysts, respectively.

The XPS Pd 3d spectra for all carbon-replicas supported catalysts are similar (Fig. S5). Two palladium states with predomination (above $86 \%$ ) of the component at $335.5 \pm 0.1$ $\mathrm{eV}$, assigned to metallic palladium, can be seen. The 9-14\% of palladium corresponds to the palladium species of higher binding energy $337.2 \pm 0.1 \mathrm{eV}$ which could be related to oxidized palladium like in the $\mathrm{PdO}$ formed as a result of surface oxidation of palladium particles. Highly dispersed $\mathrm{Pd}^{0}$ clusters are capable to re-oxidise during contact with oxygen [14], for instance, upon storage of catalysts in air. The Pd metallic assisted by an oxidized palladium species $\left(\mathrm{Pd}^{\delta+}\right)$ was also observed in the $\mathrm{Pd} / \mathrm{CNT}, \mathrm{Pd} /$ carbon black and $\mathrm{Pd} /$ active carbon samples [14, 48, 52]. Similar XPS spectra of the palladium particles in all the carbon replicas-supported catalysts are consistent with results reported by Toebes et al. [67] who observed that the varying concentrations of oxygen-containing carbon surface groups in the series of $\mathrm{Pt} / \mathrm{CNF}$ catalysts did not give rise to the shift in the $\mathrm{Pt} 4 \mathrm{f}_{7 / 2}$ binding energy. The $\mathrm{H}_{2}$ chemisorption measurements also revealed that oxygenated carbon functionalities do not affect platinum electronic properties in these $\mathrm{Pt} / \mathrm{CNF}$ catalysts.

The XPS Pd $3 \mathrm{~d}_{5 / 2}$ spectrum of the $\mathrm{Pd} / \mathrm{VC}$ catalyst also shows two palladium states at $335.6 \mathrm{eV}(61 \%)$ and $336.5 \mathrm{eV}$ (39\%) (Table 3). However, their contributions differ from those in the carbon replicas-supported catalysts. Moreover, the $\mathrm{Pd} 3 \mathrm{~d}_{5 / 2}$ binding energy of $336.5 \mathrm{eV}$ suggests modification of palladium electronic properties due to electroactive VC carbon. This effect was observed in VC-supported Pd, $\mathrm{Pt}, \mathrm{Ru}$ electrocatalysts $[68,69]$ and was ascribed to electronic effect induced by $\pi$ electrons of the graphitic planes in the $\mathrm{VC}$ carbon. For instance, the $\mathrm{Pt} 4 \mathrm{f}_{7 / 2}$ binding energy in the $\mathrm{Pt} / \mathrm{VC}$ electrocatalyst was higher than the energy characteristic of bulk platinum [68].

\subsection{Pd distribution}

Here, the catalysts are synthesized by the incorporation of $\mathrm{Pd}$ particles prepared via microemulsion method into the carbon materials, series $\mathrm{SiO}_{2}$ and SBA-15, and into VC carbon black. The same preparation procedure used in our previous work (microemulsion composition) produced the VC-supported monometallic $\mathrm{Pd}, \mathrm{Pt}, \mathrm{Ru}$, and bimetallic $\mathrm{Pd} /$ $\mathrm{Pt}, \mathrm{Pd} / \mathrm{Au} / \mathrm{Pd} / \mathrm{Ir}$ catalysts with uniformly dispersed metal nanoparticles of size within narrow range, $4-8 \mathrm{~nm}$ and the average particle size of approximately $4-5 \mathrm{~nm}$ [55].

In the present study, the XRD results indicate the Pd crystallites of the same average size, $4-5 \mathrm{~nm}$ in all the synthesized catalysts. The electron microscopy images clearly demonstrate that the structural characteristics of the carbon samples are crucial for the metal particles distribution in the final catalysts. It should be noticed that the transfer of Pd nanoparticles prepared in microemulsion (ME) to carbon support was carried out in the following way. The carbon support was added to the suspension of the metal particles in the ME medium consisting of a non-polar cyclohexane bulk phase under vigorous stirring, after which THF was slowly added in order to break the microemulsion and allow the particles to deposit onto the support. Therefore, the particle distribution throughout the carbon sample could be expected to be influenced by the morphological and structural properties of the carbon.

Figure 6 compares the SEM images of $\mathrm{SiO}_{2}$ and SBAseries catalysts and $2 \% \mathrm{Pd} / \mathrm{VC}$. The micrograph of $2 \% \mathrm{Pd} / \mathrm{VC}$ catalyst demonstrates the Pd particles of almost spherical shape which are well-dispersed throughout the entire carbon grain surface, although some small aggregates are visible. Similar morphology was previously observed in our studies for VC-supported catalysts [55, 57]. This metal distribution was ascribed to the morphology of VC carbon which enables penetration of metal particles inside the grains because of the open porosity structure of the meso/macropores existing between the carbon grains consisting of aggregated 
Fig. 6 SEM micrographs of the $\mathrm{SiO}_{2}$-series catalysts: $\mathrm{Pd} / \mathrm{C}(\mathrm{ss}-\mathrm{Si})$ and $\mathrm{Pd} / \mathrm{C}(\mathrm{ff}-\mathrm{Si})$, and SBA-series catalysts: $\mathrm{Pd} / \mathrm{C}$ (ssSBA) and Pd/(ff-SBA), as well as Vulcan XC-72 carbon black supported $\mathrm{Pd} / \mathrm{VC}$ catalyst; images in COMPO mode (white spots - palladium) with insets in SEI mode (surface morphology)
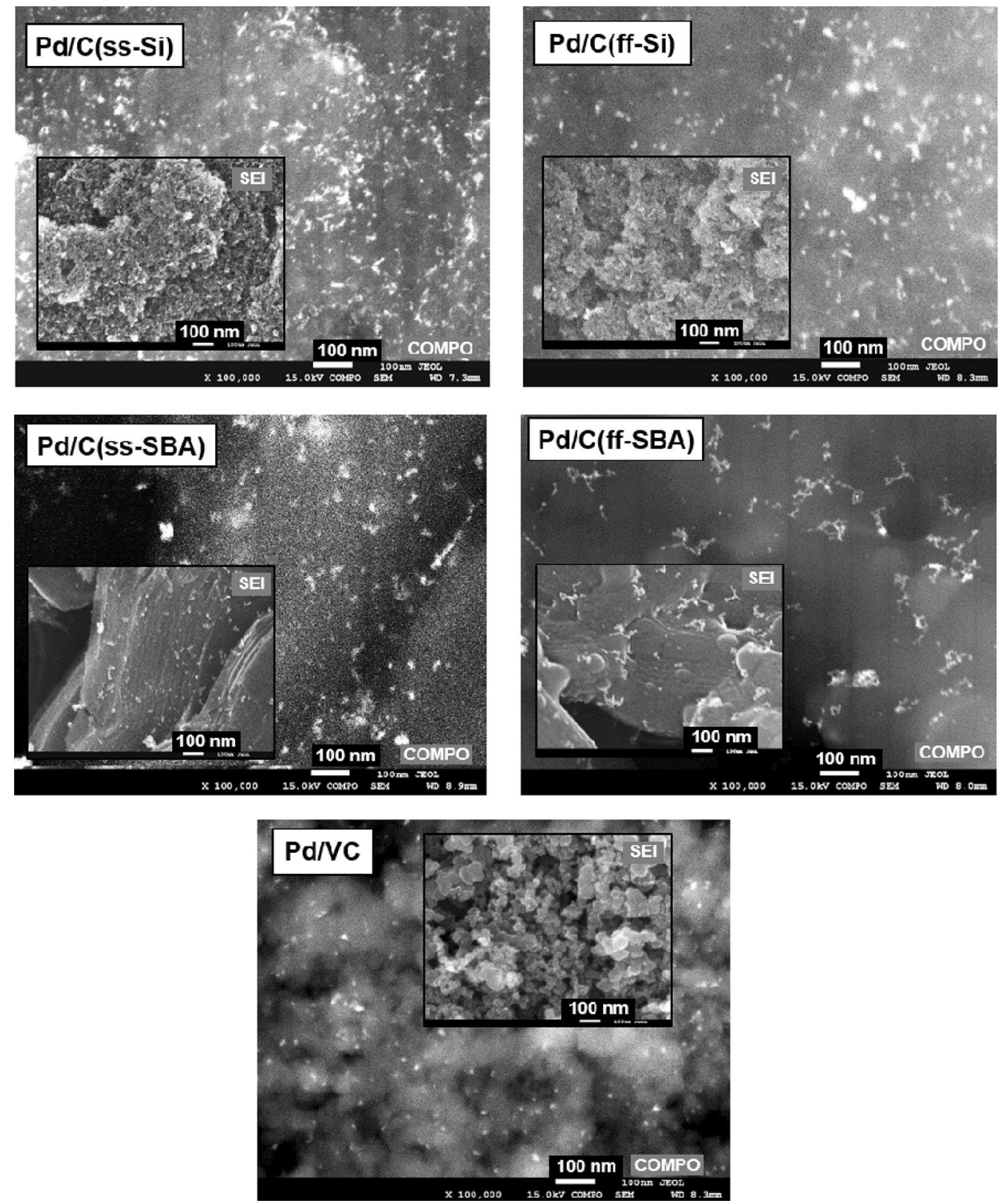

spherical primary VC particles. This is clearly observable in the HRTEM images of our VC sample (Fig. 5d). It was reported [70] that in the VC-supported $\mathrm{Pd}_{3} \mathrm{Pt}$ catalyst, the open structure of $\mathrm{VC}$ material generated between the spherical carbon particles allowed the penetration of ca. $5 \mathrm{~nm}$ sized $\mathrm{Pd}_{3} \mathrm{Pt}$ metal particles inside the bulk and location them on the surface of open pores.

In the case of $\mathrm{SiO}_{2}$-templated carbon samples, no longrange pore ordering exists but a worm-like structure is revealed. This morphology leads to relatively well-dispersed metal particles in the $\mathrm{Pd} / \mathrm{C}(\mathrm{ss}-\mathrm{Si})$ and $\mathrm{Pd} / \mathrm{C}(\mathrm{ffSi}$ ) catalysts (Fig. 6). In the $\mathrm{Pd} / \mathrm{C}$ (ss-Si) catalyst all the Pd particles are generally isolated from each other and have a narrow size distribution with only occasionally observed small aggregates composed of a few particles (Fig. 7). The particles are more aggregated in the $\mathrm{Pd} / \mathrm{C}(\mathrm{ff}-\mathrm{Si})$ catalyst where apart from the individual metal particles a number of aggregates can be seen (Fig. 6). The comparison of the SEM images for the carbon replicas supported catalysts with that of $2 \% \mathrm{Pd} /$ VC sample indicates much lower "density of white spots" in the image of the latter, although its bulk palladium loading $2 \% \mathrm{wt} \mathrm{Pd} / \mathrm{VC}$ exceeds those in the nanostructured carbonssupported catalysts (1.07-1.40 wt\% Pd, Table 3). This observation is in line with the previously discussed Pd surface concentration determined by XPS. Thus, the $\mathrm{SiO}_{2}$-series catalysts both are characterized by strongly inhibited Pd penetration resulting in a significant enrichment of outer surface in Pd.

On the other hand, there is no enrichment of outer surface in $\mathrm{Pd}$ in the $\mathrm{Pd} / \mathrm{VC}$ catalyst evidenced by almost equal $\mathrm{Pd}$ bulk and surface concentrations $\left(\mathrm{Pd}_{\mathrm{S}} / \mathrm{Pd}_{\mathrm{B}}=1.3\right.$, Table 3$)$. The SEM images show that the morphology composed of 
Fig. 7 TEM (left) and HRTEM (right) micrographs of $\mathrm{Pd} / \mathrm{C}$ (ssSi) catalyst
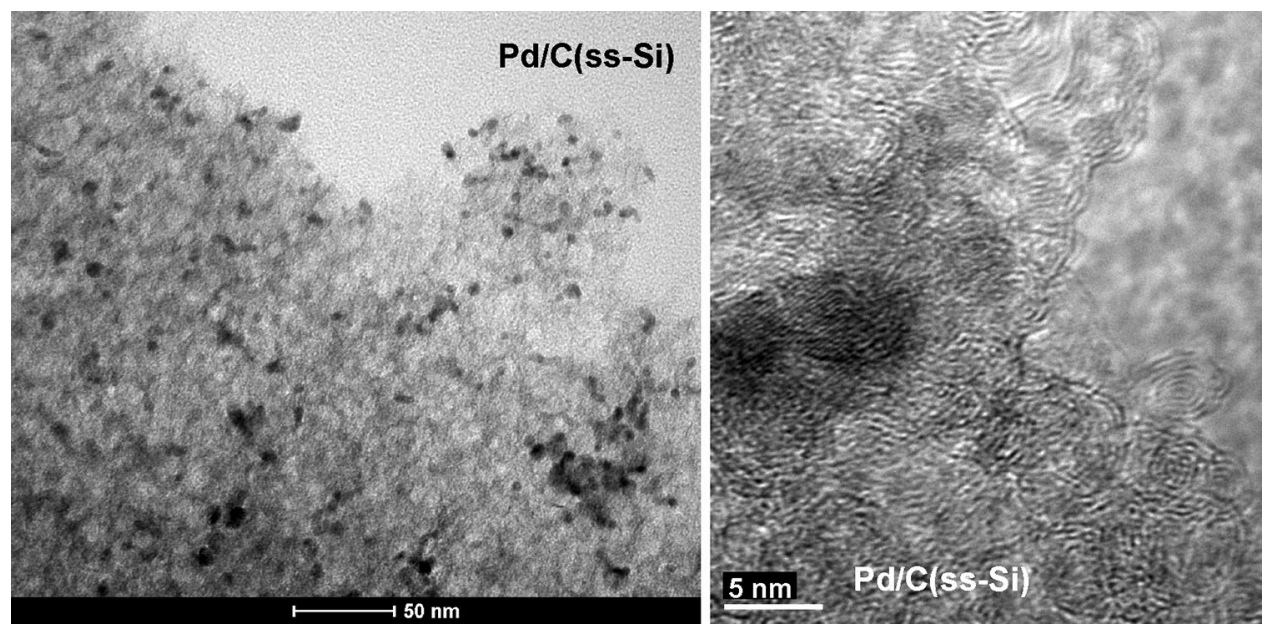

carbon nanorods in the SBA-series catalysts allowed dispersion of the particles through the supports. However, there are no individual metal particles but less or more branched structures composed of several primary Pd particles exist what is distinctly visible in the HRTEM images (Fig. 8).

Both SBA-15 derived carbons characterize by ordered pore structures with uniform mesopores of width of $2-3 \mathrm{~nm}$, much below the Pd particle size, 4-5 nm. It prevented penetration of the Pd particles inside the pore system. Location of the particles on the outer surface of the carbon nanorods blocked the entrance to some of the pores thus lowering the surface area of the Pd-containing samples, observed by BET data (Table 1). Similar effect was reported for the HMS-templated ordered mesoporous carbon supported $\mathrm{Pt}$ electrocatalysts [16].

Both these catalysts Pd/C(ss-SBA) and Pd/C(ff-SBA) have Pd-enriched surface evidenced by much higher Pdsurface concentration than the Pd-bulk $\left(\mathrm{Pd}_{\mathrm{S}} / \mathrm{Pd}_{\mathrm{B}}=16.1\right.$ and 16.8 , respectively). However, very high surface area of the SBA-templated ordered carbons accompanied by low surface
Fig. 8 TEM and HRTEM micrographs of Pd/C(ss-SBA) and $\mathrm{Pd} / \mathrm{C}(\mathrm{ff}-\mathrm{SBA})$ catalysts
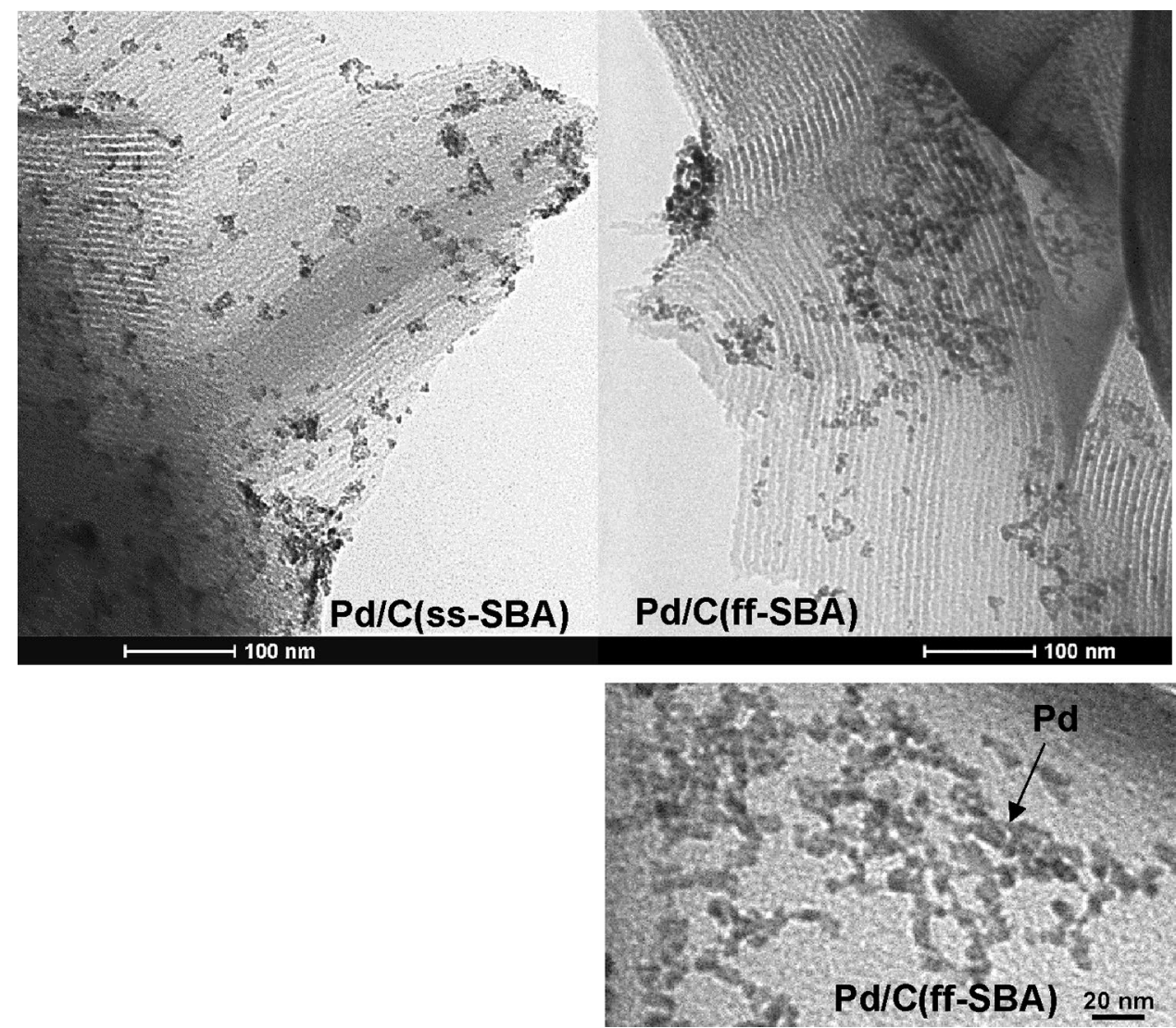
content of oxygenated carbon groups resulting in a low surface density of the oxygenated carbon groups did not effectively prevent against the metal particles migration/aggregation and various branched Pd structures could be formed (Fig. 8). Similar effect was observed in the $\mathrm{Pd}_{3} \mathrm{Pt}-\mathrm{CMK}-3$ system [70]. The size of the metal particles (ca. $5 \mathrm{~nm}$ ) was larger than the pore size of ordered nanostructured carbon CMK-3 (ca. $3 \mathrm{~nm}$ ) which resulted in distinct agglomeration of the $\mathrm{Pd}_{3} \mathrm{Pt}$ particles on the external surface of the carbon.

The nanostructured $\mathrm{SiO}_{2}$-derived carbon replicas present the mesostructure with the average pore width of ca. $8 \mathrm{~nm}$ (Fig. 3a), which are large enough to accommodate the Pd particle of $4-5 \mathrm{~nm}$ in size. As a result, both $\mathrm{Pd} / \mathrm{C}(\mathrm{ss}-\mathrm{Si})$ and $\mathrm{Pd} / \mathrm{C}$ (ff-Si) catalysts are characterized by somewhat lower enrichment of outer surface in $\mathrm{Pd}\left(\mathrm{Pd}_{S} / \mathrm{Pd}_{B}=10.7\right.$ and 10.0, respectively) when compared to their counterparts synthesized using the SBA-15 template (Table 3).

There is no difference in an enrichment of outer surface in $\mathrm{Pd}$ between the $\mathrm{Pd} / \mathrm{C}(\mathrm{ss}-\mathrm{Si}$ ) and $\mathrm{Pd} / \mathrm{C}(\mathrm{ff}-\mathrm{Si}$ ). However, the furfuryl alcohol-derived carbon replica produces the $\mathrm{Pd} / \mathrm{C}(\mathrm{ff}-\mathrm{Si}$ ) catalyst with much more aggregated Pd particles compared to the sucrose-arising $\mathrm{PdC} /(\mathrm{ss}-\mathrm{Si}$ ) sample. Both carbon samples are highly hydrophobous as they are characterized by similar and low surface content of oxygenated groups. It should be noticed that the former $\mathrm{C}(\mathrm{ff}-\mathrm{Si}$ ) posses ca. 2.5-times lower surface area and characterizes by a higher degree of graphitization than the sucrose-derived carbon. Moreover, Zhai et al. [56] observed that with the same silica template (SBA-15), sucrose precursor produced the carbon rod-like products with coarser pore walls than the furfuryl alcohol-derived sample, which could be helpful to prevent the Pd particles from agglomeration.

The VC carbon characterizes by an extended mesoporous structure, however, small mesopores with diameter below $3 \mathrm{~nm}$ dominate (Fig. 5b). The Pd particles are too large to reside within most of these mesopores. Therefore, the open morphology in form of a number of accidentally distributed meso/macropores generated between the spherically shaped carbon particles exist (Fig. 5d,f). Moreover, the surface area of $\mathrm{VC}$ sample equal to $228 \mathrm{~m}^{2} / \mathrm{g}$ is much lower than the surface area of mesostructured carbons, whereas all the carbons are of similar oxygen surface content (Table 2). It makes the surface density of oxygenated groups much higher in the case of the VC carbon which might facilitate the incorporation of hydrophilic Pd-containing micelle on the inner surface of the VC carbon grains. As a result, the Pd-surface and Pd-bulk concentrations in the $\mathrm{Pd} / \mathrm{VC}$ catalyst are comparable $\left(\mathrm{Pd}_{\mathrm{S}} / \mathrm{Pd}_{\mathrm{B}}=1.3\right.$, Table 3$)$ and the $\mathrm{Pd}$ particles aggregation is almost avoided.

In summary, the obtained results show that the type of carbon precursor affected the morphology and textural characteristic (surface area, pore volume) of the synthesized carbon replicas, consistent with previous reports [56,
71-74]. In general, irrespective of the used silica template, the obtained carbons characterize by similar and low surface content of oxygenated carbon groups. The carbon replicas prepared with sucrose precursor display higher surface area and pore volume than their counterparts synthesized using furfuryl alcohol. The influence of the carbon precursor manifests more distinctly for the $\mathrm{SiO}_{2}$-templated carbons. All the carbons are of low crystallinity; however, the furfuryl alcohol-derived carbons exhibit somewhat higher degree of graphitization. Very recently, Li et al. [75] reported that carbon source affected the hydrophobous nature of the carbon replica surface. With the same silica template the sucrosederived carbon was more hydrophobic than the anthracenederived one. With the same silica template (like SBA-15) sucrose and furfuryl alcohol produced ordered carbons with amorphous pore walls, whereas the carbon samples derived from the aromatic precursors (naphthalene, anthracene, etc.) characterized by improved graphitic character [64, 76, 77]. It has been also observed that among various carbon precursors (furfuryl alcohol, anthracene, naphthalene, petroleum pitch), the sucrose-derived carbon replicas have much higher surface area and pore volume than aromatic precursors-derived counterparts attributed to a release of volatile species $\left(\mathrm{H}_{2}\right.$, $\mathrm{CO}, \mathrm{CO}_{2}$ ) during the sucrose carbonization [56, 71].

In all our catalysts the Pd crystallites are of the same average size $4-5 \mathrm{~nm}$ and there is no essential difference in the surface content of oxygen in the carbon supports. However, the surface Pd concentration and the morphology of Pd phase manifesting by the degree of particles aggregation are different. This implies a crucial influence of the morphological and structural features of the carbon materials on the Pd phase, the Pd particles penetration inside the pore system and their aggregation. Similar effect has been described by Cabiac et al. [72] who observed that textural properties of activated carbons with a similar surface oxygen content influenced dispersion of the "in-situ" $\left(\mathrm{H}_{2} \mathrm{PdCl}_{4}\right.$, $\mathrm{NaOH}, \mathrm{H}_{2}$-reduction) generated Pd colloidal clusters. The correlation between the inner pore surface of these carbons and the Pd dispersion was observed.

Among all the synthesized catalysts, the $\mathrm{Pd} / \mathrm{C}$ (ss-Si) sample characterizes by the morphological and textural features attractive from the catalytic point of view. The $\mathrm{Pd} / \mathrm{C}(\mathrm{ss}-\mathrm{Si})$ catalyst has high surface area $\left(1167 \mathrm{~m}^{2} / \mathrm{g}\right)$ and large pore volume $\left(2.42 \mathrm{~cm}^{3} / \mathrm{g}\right)$ with very low contribution of microporosity (ca. $4 \%$ ).

\subsection{Catalytic results}

The hydrogenation tests of FU are studied in 2-propanol as the solvent. 2-propanol is applied as a hydrogen donor reagent in the catalytic transfer hydrogenation reaction $(\mathrm{CTH})$. However, we did not observe FU conversion under our 
hydrogenation conditions when nitrogen instead of hydrogen (6 bar $\mathrm{N}_{2}, 35^{\circ} \mathrm{C}$ ) was used.

The reagents distribution profiles over studied catalysts are displayed in Fig. 9. Figure 10a compares the conversions of FU in the presence of tested catalysts. It is observed that both SBA-series catalysts display similar FU conversion. As the result, initial $\mathrm{FU}$ hydrogenation rates normalized to the Pd mass are almost the same over $\mathrm{Pd} / \mathrm{C}(\mathrm{ss}-\mathrm{SBA})$ and $\mathrm{Pd} / \mathrm{C}(\mathrm{ff}-\mathrm{SBA})$ catalysts $(0.078$ and $0.076 \mathrm{~mol} \mathrm{FU} / \mathrm{min} \cdot \mathrm{gPd}$, respectively) (Table 5). There is no difference in the Pd surface concentration (Table 3 ) and in the Pd particles distribution in these two catalysts (Fig. 8). The initial rate is higher and equal to $0.093 \mathrm{~mol} \mathrm{FU/min.gPd} \mathrm{over} \mathrm{Pd/C(ss-Si)} \mathrm{cata-}$ lyst which characterizes by much better dispersed and mostly non-aggregated Pd particles (Fig. 7).

The $\mathrm{Pd} / \mathrm{C}$ (ff-Si) catalyst prepared using the $\mathrm{SiO}_{2}$ template but furfuryl alcohol precursor is less active. In its presence the reaction slows down when FU conversion reaches ca. $40 \%$ (Fig. 10a). Similar effect can be seen over the Pd/VC catalyst which is the least active one (Table 5).

The reagents distribution diagrams (Fig. 9) show that FA and THFA are the major products formed over all the tested catalysts. No tetrahydrofurfural (THFU) was detected among the products, except the traces (below 1\%) observed over the $\mathrm{Pd} / \mathrm{C}(\mathrm{ss}-\mathrm{Si}$ ) catalyst only. Thus, it might be assumed that THFA is formed through the FA hydrogenation, similarly to previous study over Pd catalysts [49, $78,79]$. The GC-MS analysis shows traces of furfuryl isopropyl ether (ET) and furfural diisopropyl acetal (AC), the products of weak acids catalysed reactions with the participation of 2-propanol. It has been observed that the protons provided by the ethanol solvent [80] or acid functional groups of carbon supports in the active carbon - Ru $[52,81]$ and in the MWNT - Pd, Pt [82] catalysed the formation of AC and ET products. In the presence of all SBAand $\mathrm{SiO}_{2}$-series catalysts very low amount of ET is formed only above $50 \% \mathrm{FU}$ conversion, attaining up to $2 \mathrm{~mol} \%$ in final solution. Similarly, only the traces of AC are detected over the $\mathrm{SiO}_{2}$-series catalysts (Fig. 9). However, over the $\mathrm{Pd} / \mathrm{VC}$ catalyst $\mathrm{ET}$ is observed from the very beginning of the reaction with the amount comparable to that of THFA. It should be noticed that other side products termed as "humins" including the products of FA oligomerization, self-etherification as well as FU degradation, etc. could be also formed [83]. For instance, the level of side products attained $40-60 \%$ over Pd/active carbon under similar conditions (2-propanol, $30{ }^{\circ} \mathrm{C} .3$ bar $\mathrm{H}_{2}$ ) [48], $12 \%$ over $\mathrm{Pd} / \mathrm{MWNT}$ [82], $13 \%$ on Pd/active carbon [38] and 5-13\% on $\mathrm{Pd} / \mathrm{SiO}_{2}[78]$ catalysts. Figure 9 shows the carbon mass balance curves $(\mathrm{CB})$ calculated as the sum of all identified products: $\mathrm{FA}+\mathrm{THFA}+\mathrm{AC}+\mathrm{ET}$. In the presence of all our catalysts, the mass balance is above $90 \%$, achieving 92-95\% over both SBA-series catalysts up to the maximum content of FA. When the FA content passes over the maximum, the amount of by-products slowly increases reaching ca. $15-20 \%$ in final solutions. Similar trends can be seen over all studied catalysts.

As shown in Fig. 9, furfuryl alcohol dominates among the products formed over all tested catalysts. At FU conversion approaching 50\%, the comparable selectivities to FA, 51 and $54 \%$ are attained over both SBA-series catalysts, however they are lower than the selectivity of $65 \%$ obtained over the most active $\mathrm{Pd} / \mathrm{C}$ (ss-Si) catalyst (Fig. 10b; Table 5). The FA selectivity is lower, ca. 42-43\% over much less active $\mathrm{Pd} / \mathrm{C}(\mathrm{ff}-\mathrm{Si}$ ) and $\mathrm{Pd} / \mathrm{VC}$ catalysts. The catalysts display also different selectivity to tetrahydrofurfuryl alcohol (THFA) which is the consecutive product of FA hydrogenation (Fig. 10c). The THFA selectivities over the SBA-series catalysts are $12-13 \%$ (at 50\% FU conversion) being higher than the selectivity of 6-7\% observed over the $\mathrm{SiO}_{2}$-series catalysts and $\mathrm{Pd} / \mathrm{VC}$ sample (Table 5).

As Fig. 10b shows, the content of FA reaches a maximum and starts to decrease as the result of its sequential hydrogenation to form THFA and/or other products. In the presence of the least active $\mathrm{Pd} / \mathrm{C}(\mathrm{ff}-\mathrm{Si}$ ) and $\mathrm{Pd} / \mathrm{VC}$ catalysts, the reaction slows down before the maximum yield of FA is achieved.

Similar effect presenting a gradual decrease of FU hydrogenation rate over $\mathrm{Pd} / \mathrm{CNT}$ and $\mathrm{Pd} / \mathrm{CB}$ (carbon black) catalysts observed under mild reaction conditions $\left(50-90{ }^{\circ} \mathrm{C}\right.$, $0.5-2 \mathrm{MPa}$ ) has been related to a blockage of active sites by
Table 5 Catalytic data of furfural hydrogenation (Reaction conditions: 2-propanol, FU concentration $0.05 \mathrm{~mol} / \mathrm{dm}^{3}, 35^{\circ} \mathrm{C}, 6$ bar $\mathrm{H}_{2}$, catalyst concentration $0.7 \mathrm{~g} /$ $\mathrm{dm}^{3}$ )

\begin{tabular}{|c|c|c|c|c|c|c|}
\hline \multirow[t]{2}{*}{ Catalyst } & \multirow[t]{2}{*}{$\begin{array}{l}\text { Initial rate } \\
(\mathrm{mol} / \mathrm{min} \cdot \mathrm{g} \mathrm{Pd})\end{array}$} & \multicolumn{2}{|c|}{$\begin{array}{l}\text { Selectivity at } 50 \% \\
\text { FU conversion }(\%)\end{array}$} & \multicolumn{3}{|c|}{ Yield (\%) } \\
\hline & & FA & THFA & $\begin{array}{l}\text { FA } \\
\text { Max }\end{array}$ & $\begin{array}{l}\text { THFA } \\
\text { at max } \\
\text { yield FA }\end{array}$ & $\begin{array}{l}\mathrm{FA}+\mathrm{THFA} \\
\text { at max yield FA }\end{array}$ \\
\hline $\mathrm{Pd} / \mathrm{C}(\mathrm{ss}-\mathrm{Si})$ & 0.093 & 65 & 7.0 & 52 & 15.5 & 67.5 \\
\hline $\mathrm{Pd} / \mathrm{C}(\mathrm{ff}-\mathrm{Si})$ & 0.023 & 43 & 7.2 & & & \\
\hline $\mathrm{Pd} / \mathrm{C}$ (ss-SBA) & 0.078 & 54 & 11.8 & 43 & 17 & 60 \\
\hline $\mathrm{Pd} / \mathrm{C}(\mathrm{ff}-\mathrm{SBA})$ & 0.076 & 51 & 13.2 & 34 & 26 & 60 \\
\hline $\mathrm{Pd} / \mathrm{VC}$ & 0.017 & 42 & 6.5 & & & \\
\hline
\end{tabular}


Fig. 9 Reagents distribution profile obtained in the hydrogenation of furfural over Pd/C(ss-Si), Pd/C(ff-Si), $\mathrm{Pd} / \mathrm{C}$ (ss-SBA), Pd/C(ff-SBA) and $\mathrm{Pd} / \mathrm{VC}$ catalysts; Reaction conditions: 2-propanol, $35^{\circ} \mathrm{C}$, 6 bar $\mathrm{H}_{2}$, furfural concentration $0.05 \mathrm{~mol} / \mathrm{dm}^{3}$, catalyst concentration $0.71 \mathrm{~g} / \mathrm{dm}^{3}$
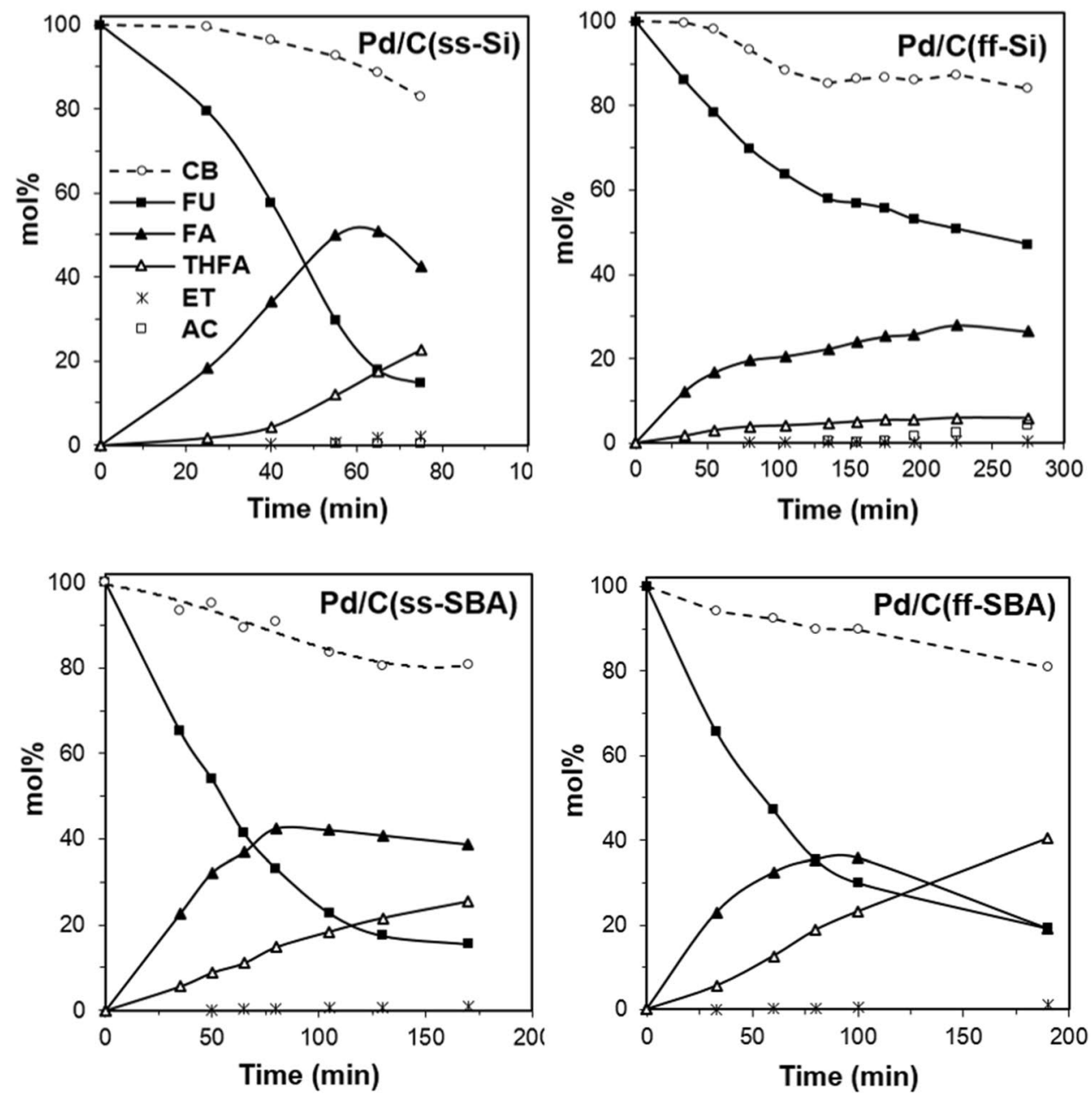

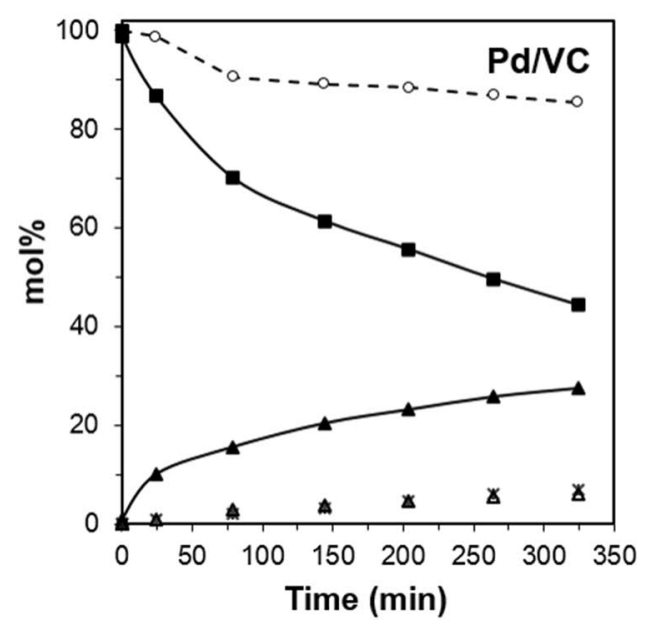

reaction products, among them FA [52]. However, the other possibility could be the presence of "humins" formed by FA polymerization or strong adsorption of carbon monoxide (CO) formed as a product of FU decarbonylation.

A partial blockage of active sites in the $\mathrm{Pd} / \mathrm{SiO}_{2}$ catalysts has been established by Nakagawa et al. [78] to be a result of a considerably stronger adsorption of FA than
FU (reaction in aqueous phase, $2{ }^{\circ} \mathrm{C}, 8 \mathrm{MPa}$ ). The FA adsorption was much stronger than that of $\mathrm{FU}$ in the $\mathrm{Pd} /$ $\mathrm{SiO}_{2}$ with larger Pd crystallite of $7 \mathrm{~nm}$ and the activity decreased since the very beginning of the test. On the other hand, comparable adsorption equilibrium constants for FA and $\mathrm{FU}$ were calculated in the case of $\mathrm{Pd} / \mathrm{SiO}_{2}$ catalyst with fine $\mathrm{Pd}(2 \mathrm{~nm})$ crystallites and there was no 
Fig. 10 Furfural FU conversion vs. reaction time (a), the amount of produced furfuryl alcohol FA (b) and tetrahydrofurfuryl alcohol THFA (c) over Pd/C(ss$\mathrm{Si}), \mathrm{Pd} / \mathrm{C}(\mathrm{ff}-\mathrm{Si}), \mathrm{Pd} / \mathrm{C}$ (ss-SBA), $\mathrm{Pd} / \mathrm{C}(\mathrm{ff}-\mathrm{SBA})$ and $\mathrm{Pd} / \mathrm{VC}$ catalysts; Reaction conditions: 2-propanol, $35^{\circ} \mathrm{C}, 6$ bar $\mathrm{H}_{2}$, furfural concentration $0.05 \mathrm{~mol} /$ $\mathrm{dm}^{3}$, catalyst concentration 0.71 $\mathrm{g} / \mathrm{dm}^{3}$
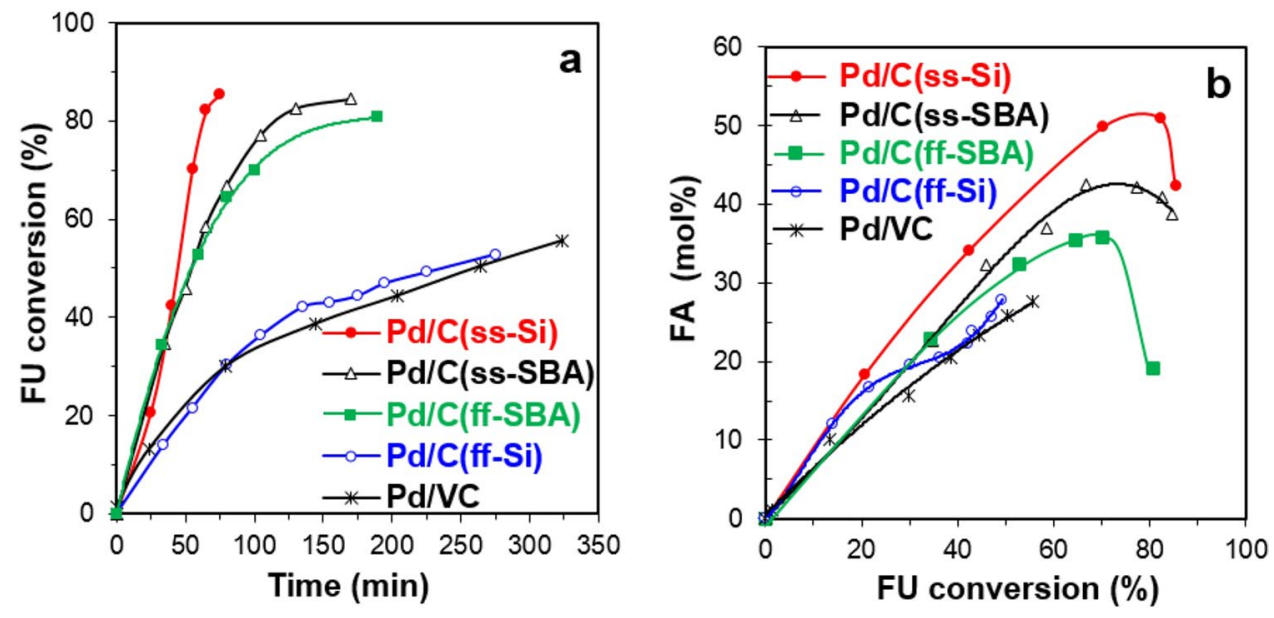

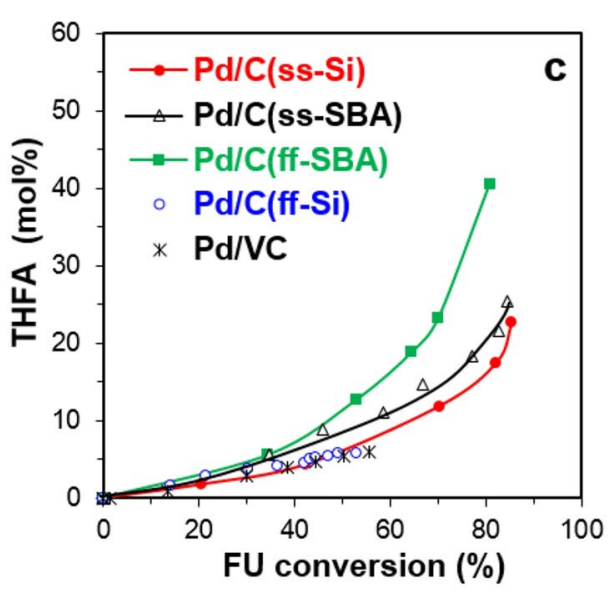

blockage of active sites observed. This shows, according to the authors, that the Pd crystal size affected the adsorption of FA relative to that of FU, being weaker in the case of smaller Pd particles.

It has been also observed that the $\mathrm{Pd} / \mathrm{C}$ catalyst which has the Pd particles inside the pores of modified carbon material formed the Pd microenvironment with $\mathrm{C}-\mathrm{OH}$ and $\mathrm{COOH}$ groups which significantly affected phenol hydrogenation. Phenol, being a strongly H-bridge forming reagent was enriched in the pores leading to distinctly separated reaction stages, the hydrogenation of aromatic ring followed by the reduction of the $\mathrm{C}-\mathrm{OH}$ [84]. An influence of oxygenated carbon groups on the hydrogenation of $\alpha, \beta$-unsaturated aldehydes (cinnamaldehyde) has been observed over Pd/C [85], Pt/CNF [67] and Ru/CNF [86] catalysts. The presence of oxygenated groups in the carbon surface/metal particle interfaces caused some changes in the activity/selectivity through modification of the reagents adsorption.

In the case of our catalysts, the Pd metal crystallites are of similar size and no essential difference in the palladium state was observed by XPS. However, the applied carbon supports can provide a specific microenvironment for the metal sites, such as functional oxygenated carbon groups, observed by XPS and FT-IR. Their presence in close proximity to the $\mathrm{Pd}$ sites in the metal nanoparticles (differently aggregated as well as more or less deeply located in the porous carbon structure) can exert the hydrogenation reaction.

In our catalysts there is no essential difference in the surface content of oxygen in the carbon supports. However, the surface Pd concentration and the morphology of the Pd phase manifesting by the degree of particles aggregation are different. Thus, in discussion of activity/selectivity effects, we introduced the oxygen to palladium atomic ratio $(\mathrm{O} / \mathrm{Pd}$ parameter) calculated from the XPS results (Table 3). The contents of FA and THFA against the conversion of FU are compared in Fig. 10b. It can be seen that over the Pd/C(ff$\mathrm{Si}$ ) catalyst with the highest $\mathrm{O} / \mathrm{Pd}=10.1$ (Table 3 ) as well as over the $\mathrm{Pd} / \mathrm{VC}$ catalyst with the deeply located Pd particles the reaction slows down from the its beginning. This effect could be the most probably related to a partial and slow blockage of active site due to the FA adsorption facilitated by a growing tendency to $\mathrm{H}$-bond formation with the participation of the surface $\mathrm{C}-\mathrm{OH}$ and/or $\mathrm{COOH}$ groups. 
According to FTIR spectra (Fig. S1) of the Pd/C(ffSi) and $\mathrm{Pd} / \mathrm{C}(\mathrm{ss}-\mathrm{Si})$ catalysts before and after the catalytic tests, there are no remarkable changes in the spectrum of the $\mathrm{Pd} / \mathrm{C}$ (ss$\mathrm{Si}$ ) catalyst after the hydrogenation test. On the other hand, in the spectrum of spent $\mathrm{Pd} / \mathrm{C}(\mathrm{ff}-\mathrm{Si}$ ) catalyst, besides some changes in the $\mathrm{C}-\mathrm{O}$ region, new bands appear in the range $2800-3000 \mathrm{~cm}^{-1}$, characteristic of the $\mathrm{C}-\mathrm{H}$ vibrations in the $-\mathrm{CH}_{2},-\mathrm{CH}_{3}$ groups. This could be an evidence that some organic species remain in the spent $\mathrm{Pd} / \mathrm{C}(\mathrm{ff}-\mathrm{Si})$ catalyst.

It can be seen (Table 3 ) that high Pd surface concentration is accompanied by a low $\mathrm{O} / \mathrm{Pd}$ ratio in both SBA-series catalysts and in the $\mathrm{Pd} / \mathrm{C}(\mathrm{ss}-\mathrm{Si})$ one. All three catalysts offer high rate of the FU hydrogenation (Table 5) and the sum of FA and THFA yields (calculated at the maximum FA yield) attains a high level of $60-67 \%$. However, the surface O/Pd values are the lowest in the $\mathrm{Pd} / \mathrm{C}$ (ff-SBA) and $\mathrm{Pd} / \mathrm{C}$ (ss-SBA) catalysts ( 3.55 and 5.13 , respectively, Table 3 ), both characterize by the high surface concentration of palladium which exists in the form of various branched structures composed of the $\mathrm{Pd}$ particles. The $\mathrm{O} / \mathrm{Pd}$ ratio equal to 5.7 is somewhat higher in the case of $\mathrm{Pd} / \mathrm{C}(\mathrm{ss}-\mathrm{Si}$ ) catalyst with well-dispersed Pd particles. As Fig. 10b shows, the difference in the maximum content of FA observed on these catalysts seems to be a result of various tendencies to the $\mathrm{C}=\mathrm{C}$ activation producing THFA. The tendency is higher over both SBA-series catalyst being the highest in the case of the $\mathrm{Pd} / \mathrm{C}(\mathrm{ff}-\mathrm{SBA})$ catalyst evidenced by the FA maximum yield of $34 \%$ attained at ca. $65 \%$ FU conversion (Table 5). The higher maximum yield of $43 \%$ over the $\mathrm{Pd} / \mathrm{C}$ (ss-SBA) is observed and it is attained at somewhat higher FU conversion (ca. 70\%). The maximum FA yield is the highest over $\mathrm{Pd} / \mathrm{C}(\mathrm{ss}-\mathrm{Si})$ catalyst and is observed at ca. $80 \% \mathrm{FU}$ conversion. These effects are in line with the THFA contents (Fig. 10c) and evidenced by the THFA yield of $26 \%$ over Pd/C(ff-SBA) which is higher than $15.5 \%$ observed over $\mathrm{Pd} / \mathrm{C}$ (ss- $\mathrm{Si}$ ) catalyst (Table 5). It seems to be difficult to decide if the observed difference in the FA/ THFA selectivity resulting mostly due to various tendencies in the $\mathrm{C}=\mathrm{C}$ activation is the result of the $\mathrm{Pd}$ phase properties and/or an influence of oxygenated carbon groups. It might be expected that the effect of oxygenated carbon groups will be lower in both SBA-series catalysts because of lower O/ $\mathrm{Pd}$ molar ratios and the existence of more aggregated $\mathrm{Pd}$ particles. On the other hand, in view of previous observations, the larger Pd particles exhibited higher tendency to the FA adsorption.

The Pd/C(ff-SBA) and Pd/C(ss-Si) catalysts are characterized by low $\mathrm{O} / \mathrm{Pd}$ surface ratio and their reusability in the FU hydrogenation has been studied. After the first hydrogenation run, the catalyst was washed with acetone and tested in the FU hydrogenation carried out under the same conditions as in the initial run. The conversion obtained in the first and second catalytic runs over $\mathrm{Pd} / \mathrm{C}(\mathrm{ss}-\mathrm{Si})$ and $\mathrm{Pd}(\mathrm{ff}-\mathrm{SBA})$ catalysts are compared in Fig. 11 and the corresponding

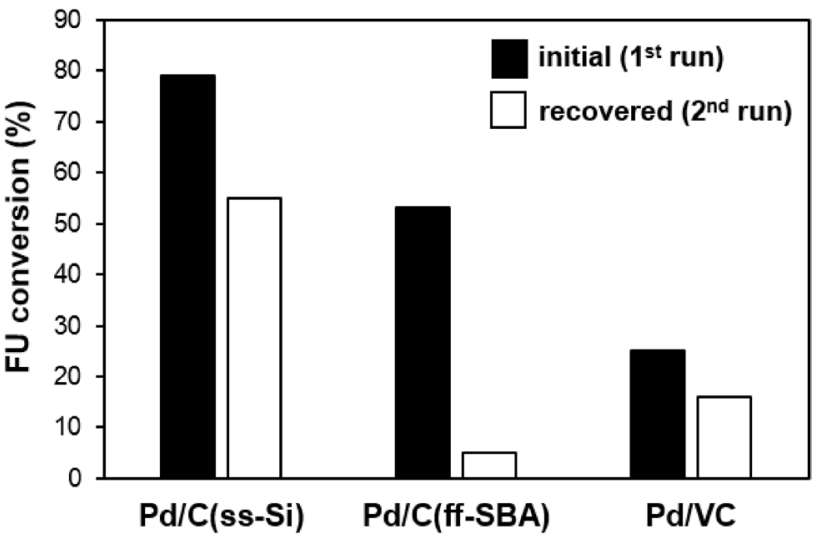

Fig. 11 The comparison of FU conversion (after $1 \mathrm{~h}$ of reaction) of initial (1st run) and recovered (2nd run) $\mathrm{Pd} / \mathrm{C}(\mathrm{ss}-\mathrm{Si}$ ), $\mathrm{Pd} / \mathrm{C}(\mathrm{ff}-\mathrm{SBA})$ and $\mathrm{Pd} / \mathrm{VC}$ catalysts; Reaction conditions: 2-propanol, $35^{\circ} \mathrm{C}, 6$ bar $\mathrm{H}_{2}$, furfural concentration $0.05 \mathrm{~mol} / \mathrm{dm}^{3}$, catalyst concentration 0.71 $\mathrm{g} / \mathrm{dm}^{3}$

selectivity results are provided in Fig. S6. The reusability of the $\mathrm{Pd} / \mathrm{VC}$ catalyst is also reported in Fig. 11 . The activity of $\mathrm{Pd} / \mathrm{C}(\mathrm{ss}-\mathrm{Si})$ in the second catalytic run is slightly lower than the initial one (Fig. 11a) evidenced by a FU conversion decrease from 79 to $55 \%$. No essential change in the products distribution is observed, although the maximum FA selectivity of $63 \%$ in the second run exceeds the $52 \%$ obtained in initial run. In contrast, a significant drop in the FU conversion can be seen for both $\mathrm{Pd} / \mathrm{C}(\mathrm{ff}-\mathrm{SBA})$ and $\mathrm{Pd} /$ $\mathrm{VC}$ catalysts. The decrease of $\mathrm{Pd} / \mathrm{C}$ (ff-SBA) activity by ca. $95 \%$ is especially distinct and is much higher compared to the activity decrease by $35 \%$ for the Pd/VC catalyst (Fig. 11). However, the SEM images registered for the spent $\mathrm{Pd} / \mathrm{C}$ (ffSBA) catalyst (Fig. S7) does not remarkably differ from that for initial catalyst (Fig. 6). The number of branched Pd structures can be seen in the spent catalyst. Therefore, the observed strong decrease of the $\mathrm{Pd} / \mathrm{C}(\mathrm{ff}-\mathrm{SBA})$ catalyst activity seems to be rather associated with a blockage effect than with a loss of metal phase.

\section{Conclusions}

Two series of mesoporous carbons were synthesized through a hard-templating method using the SBA-15 or disordered mesoporous silica gel as the templates and furfuryl alcohol or sucrose as the carbon sources. All the synthesized carbon replicas characterized by low crystallinity and both sucrosederived carbons displayed higher surface area and mesopore volume than furfuryl alcohol-derived counterparts. Employing of microemulsion procedure allowed us to prepare the Pd catalysts of similar Pd loading (1.1-1.4 wt\% Pd) and the 4-5 nm sized Pd particles. An influence of the carbon porous structure manifested by different characteristics of 
the Pd phase, such as enrichment of the surface by Pd, the surface distribution and extent of the Pd particles aggregation. The Pd catalysts supported on the SBA-15 templated carbons exhibited the highest enrichment of the surface in Pd. However, the high surface area accompanied by a low surface content of oxygenated carbon species did not effectively prevent the Pd particles aggregation. The branched Pd-structures composed of individual particles dominated in the catalysts. The enrichment of the surface in Pd was somewhat lower in the $\mathrm{SiO}_{2}$-series catalysts, but the catalysts exhibited well-dispersed Pd nanoparticles, especially the $\mathrm{Pd} / \mathrm{C}$ (ss-Si) one. The hydrogenation of furfural was studied as a probe reaction to compare catalytic properties of the $\mathrm{Pd}$ sites formed in the synthesized $\mathrm{Pd} / \mathrm{C}$ catalysts. In furfural hydrogenation (carried out at $35^{\circ} \mathrm{C}$ under 6 bar $\mathrm{H}_{2}$ pressure in 2-propanol solvent) furfuryl alcohol and tetrahydrofurfuryl alcohol were the major products formed over all the tested catalysts. The observed catalytic reactivity has been related to the properties of the Pd phase including surface Pd concentration and the extent of the metal particles aggregation accompanied by the nearest palladium environment expressed by the surface $\mathrm{O} / \mathrm{Pd}$ atomic ratio. At high $\mathrm{O} / \mathrm{Pd}$ ratio in the $\mathrm{Pd} / \mathrm{C}(\mathrm{ff}-\mathrm{Si})$ catalyst, the reaction slows down because of a facilitated furfuryl alcohol adsorption resulting most probably in a blockage of the active sites. The $\mathrm{Pd} / \mathrm{C}$ (ss$\mathrm{Si}$ ) catalyst with relatively low $\mathrm{O} / \mathrm{Pd}$ ratio and well-dispersed Pd particles exhibited the highest and most stable activity for furfural hydrogenation. The SBA-series catalysts with lower $\mathrm{O} / \mathrm{Pd}$ but more aggregated $\mathrm{Pd}$ particles exhibited lower activity but somewhat higher tendency to the tetrahydrofurfuryl alcohol formation.

Acknowledgements This research was funded in part by the statutory research fund of ICSC PAS (Jerzy Haber Institute of Catalysis and Surface Chemistry, Polish Academy of Sciences). The research was partially carried out using equipment purchased thanks to the financial support of the European Regional Development Fund in the framework of the Polish Innovation Economy Operational Program [Contract No. POIG.02.01.00-12-023/08].

\section{Compliance with ethical standards}

Conflict of interest All authors declare that they have no conflict of interest.

Open Access This article is licensed under a Creative Commons Attribution 4.0 International License, which permits use, sharing, adaptation, distribution and reproduction in any medium or format, as long as you give appropriate credit to the original author(s) and the source, provide a link to the Creative Commons licence, and indicate if changes were made. The images or other third party material in this article are included in the article's Creative Commons licence, unless indicated otherwise in a credit line to the material. If material is not included in the article's Creative Commons licence and your intended use is not permitted by statutory regulation or exceeds the permitted use, you will need to obtain permission directly from the copyright holder. To view a copy of this licence, visit http://creativecommons.org/licenses/by/4.0/.

\section{References}

1. Y. Yang, K. Chiang, N. Burke, Catal. Today 178, 197 (2011)

2. J. Lee, S. Han, T. Hycon, J. Mater. Chem. 14, 478 (2004)

3. Ch Liang, Z. Li, Sh Dai, Angew. Chem. Int. Ed. 47, 3696 (2008)

4. H. Chang, S.H. Joo, C. Pak, J. Mater. Chem. 17, 3078 (2007)

5. L. Li, Z.H. Zhu, G.Q. Lu, Z.F. Yan, S.Z. Qiao, Carbon 45, 11 (2007)

6. Y. Ding, X. Li, H. Pan, P. Wu, Catal. Lett. 144, 268 (2014)

7. J. Hu, Y. Ding, H. Zhang, P. Wu, X. Li, RSC Adv. 6, 3235 (2016)

8. B. Li, X. Li, H. Wang, P. Wu, J. Mol. Catal. A 345, 81 (2011)

9. Y. Ding, X. Li, B. Li, H. Wang, P. Wu, Catal. Commun. 28, 147 (2012)

10. L.-J. Liu, H.-M. Guo, B. Xue, H. Lou, M. Chen, RSC Adv. 5, $66704(2015)$

11. X. Li, W.-X. Chen, J. Zhao, W. Xing, Z.-D. Xu, Carbon 43, 2168 (2005)

12. F. Coloma, A. Sepulveda-Escribano, J.G. Fierro, F. RodriguezReinoso, Appl. Catal. A 150, 165 (1997)

13. D.J. Suh, T.-J. Park, Carbon 31, 427 (1993)

14. V.Z. Radkevich, T.L. Senko, K. Wilson, L.M. Grishenko, A.N. Zaderko, V.Y. Diyuk, Appl. Catal. A 335, 241 (2008)

15. W.Ch. Choi, S.I. Woo, M.K. Jeon, J.M. Sohn, M.R. Kim, H.J. Jeon, Adv. Mater. 17, 446 (2005)

16. D. Banham, F. Feng, K. Pei, S. Ye, V. Birss, J. Mater. Chem. A 1, 2812 (2013)

17. J. Ding, K.-Yu Chan, J. Ren, F.-S. Xiao, Electroch. Acta 50, $3131(2005)$

18. S.H. Liu, R.F. Lu, S.J. Huang, A.Y. Lo, S.H. Chien, S.B. Liu, Chem. Commun. 28, 3435 (2006)

19. S.H. Liu, W.Y. Yu, Ch.H. Chen, A.Y. Jo, B.J. Hwang, S.H. Chien, S.B. Liu, Chem. Mater. 20, 1622 (2008)

20. M. Inagaki, M. Kato, T. Morishita, K. Morita, K. Mizuuchi, Carbon 45, 1121 (2007)

21. B. Xu, L. Peng, G.Q. Wang, G.P. Cao, F. Wu Carbon 48, 2377 (2010)

22. Y.H. Ng, S. Ikeda, T. Harada, S. Park, T. Sakata, H. Mori, M. Matsumura, Chem. Mater. 20, 1154 (2008)

23. L.M. Shi, J.F. Yao, J.L. Jiang, L.X. Zhang, N.P. Xu, Micropor. Mesopor. Mat. 122, 294 (2009)

24. A.B. Fuertes, D.M. Nevskaia, Micropor. Mesopor. Mat. 62, 177 (2003)

25. L. Lin, P. Wang, S. Wang, Y. Zhu, B. Zhao, Y. Xie, Carbon 44, 3120 (2006)

26. L. Geng, Y. Wang, G. Yu, Y. Zhu, Catal. Commun. 13, 26 (2011)

27. Y. Wang, L. Lin, B.S. Zhu, Y.X. Zhu, Y.C. Xie, Appl. Surface Sci. 254, 6560 (2008)

28. Y. Wang, G. Yu, B. Cai, Y.X. Zhu, Y.-Ch Xie, Acta Phys. Chim. Sin. 27, 72 (2011)

29. S. Chen, R. Wojcieszak, F. Dumeignil, E. Marceau, S. Royer, Chem. Rev. 118, 11023 (2018)

30. M. Boutonnet, S. Logdberg, E.E. Svensson, Curr. Opin. Colloid \& In. 13, 270 (2008)

31. A.M. Perez-Coronado, L. Calvo, N. Alonso-Morales, F. Heras, J.J. Rodrigues, M.A. Gilarranz, Colloid. Surface A 497, 28 (2016)

32. J. Bedia, J. Lemus, L. Calvo, J.J. Rodriguez, M.A. Gilarranz, Colloid. Surface A 525, 77 (2017)

33. M. Sanchez-Dominguez, K. Pemartin, M. Boutonnet, Curr. Opin. Colloid In. 17, 297 (2012)

34. H.H. Ingelsten, J.-Ch Beziat, K. Bergvist, A. Palmqvist, M. Skoglundh, H. Quiuhong, L.K.L. Falk, K. Holmerg, Langmuir 18, 1811 (2002)

35. A. Martinez, G. Prieto, Catal. Commun. 8, 1479 (2007) 
36. R. Kosydar, M. Goral, J. Gurgul, A. Drelinkiewicz, Catal. Commun. 22, 58 (2012)

37. Y. Nakagawa, M. Tamura, K. Tomishige, ACS Catal. 3, 2655 (2013)

38. Y. Wang, P. Prinsen, K.S. Trianafyllids, S.A. Karakoulia, P.N. Trikalitis, A. Yepez, Ch Len, R. Luque, ACS Sustain. Chem. Eng. 6, 9831 (2018)

39. K. Yan, G. Wu, T. Lafleur, C. Jarvis, Renew. Sustain. Energy Rev. 38, $663(2014)$

40. V. Montes, J.F. Minambres, A.N. Khalilov, M. Boutonnet, J.M. Marinas, F.J. Urbano, A.M. Maharramov, A. Marinas, Catal. Today 306, 89 (2018)

41. D.J. Suh, T.J. Park, S.K. Ihm, Carbon 31, 427 (1993)

42. V.Z. Radkevich, T.L. Senko, K. Wilson, L.M. Grishenko, A.N. Zaderko, V.Y. Diyuk, Appl. Catal. A 335, 241 (2008)

43. S.M. Rogers, C.R.A. Catlow, C.E. Chan-Thaw, A. Chutia, N. Jian, R.E. Palmer, M. Perdjon, A. Thetford, N. Dimitratos, A. Villa, P.P. Wells, ACS Catal. 7, 2266 (2017)

44. S. Bhogeswararao, D. Srinivas, J. Catal. 327, 65 (2015)

45. Ch Li, G. Xu, X. Liu, Y. Zhang, Y. Fu, Ind. Eng. Chem. Res. 56, $8843(2017)$

46. N.S. Biradar, A.M. Henge, S.N. Birajdar, P.S. Niphadkar, P.N. Joshi, Ch.V. Rode, ACS Sustain. Chem. Eng. 2, 272 (2014)

47. R. Pizzi, R.-J. van Putten, H. Brust, S. Perathoner, G. Centi, J.C. van der Waal, Catalysts 5, 2244 (2015)

48. R. Albilali, M. Douthwaite, Q. He, S. H. Taylor Catal. Sci. Technol. 8, 252 (2018)

49. Ch Nguyen-Huy, J.S. Kim, S. Yoon, E. Yang, J.H. Kwak, M.S. Lee, K. An, Fuel 226, 607 (2018)

50. Y. Chai, S. Liu, Z.-J. Shao, J. Gong, W. Dai, G. Wu, N. Guan, L. Li, ACS Catal. 8, 8578 (2018)

51. L.-J. Liu, H.-M. Guo, B. Xue, H. Lou, M. Chen RSC Adv. 25, 66704 (2015)

52. R.M. Mironenko, O.B. Belskaya, T.I. Gulyaeva, A.I. Nizovskii, A.V. Kalinkin, V.I. Bukhtiyarov, A.V. Lavrenov, V.A. Likholobov, Catal. Today 249, 145 (2015)

53. P. Niebrzydowska, R. Janus, P. Kuśtrowski, S. Jarczewski, A. Wach, A.M. Silvestre-Albero, F. Rodriguez-Reinoso, Carbon 64, $252(2013)$

54. S. Utgenannt, F. Hansen, O. Klepel, S. Jarczewski, A. Wach, P. Kustrowski, Catal. Today 249, 38 (2015)

55. T. Szumełda, A. Drelinkiewicz, R. Kosydar, J. Gurgul, Appl. Catal. A 487, 1 (2014)

56. Y. Zhai, Y. Wan, Y. Cheng, Y. Shi, F.S. Zhang, B. Tu, D. Zhao, J. Porous Mater. 15, 601 (2008)

57. T. Szumełda, A. Drelinkiewicz, R. Kosydar, M. Góral-Kurbiel, J. Gurgul, D. Duraczyńska, Colloids Surf. A Physicochem. Eng. Asp 529, 246 (2017)

58. M.H.M.T. Assumpção, R.F.B. De Souza, D.C. Rascio, J.C.M Silva, M.L. Calegaro, I. Gaubeur, T.R.L.C. Paixão, P. Hammer, M.R.V. Lanza, M.C. Santos, Carbon 49, 2842 (2011)

59. M. Carmo, A.R. dos Santos, J.G.R. Poco, M. Linardi, J. Power Sources 173, 860 (2007)
60. R. Sellin, J.M. Clacens, Ch. Coutanceau, Carbon 48, 2244 (2010)

61. M.X. Wang, H.F. Sun, N. Ogbeifun, F. Xu, E.A. Stach, J. Xie, Mater. Chem. Phys. 123, 761 (2010)

62. S. Tang, L. Sui, Z. Dai, Z. Zhu, H. Huang, RSC Adv. 5, 43164 (2015)

63. H. Darmstadt, Ch Roy, S. Kaliaguine, T.-W. Kim, R. Ryoo, Chem. Mater. 15, 3300 (2003)

64. H. Darmstadt, C. Roy, S. Kaliaguine, S.J. Choi, R. Ryoo, Carbon 40, 2673 (2002)

65. S.M.S. Kumar, J.S. Herrero, S. Irusta, K. Scott, J. Electroanal. Chem. 647, 211 (2010)

66. L. Calvo, M.A. Gilarranz, J.A. Casas, A.F. Mohedano, J.J. Rodrigues, Ind. Eng. Chem. Res. 44, 6661 (2005)

67. M.L. Toebes, Y. Zhang, J. Hajek, T.A. Nijhuis, J.H. Bitter, A. Jos, D. van Dillen, D.C. Yu. Murzin, K.P. Koningsberger, de Jong, J. Catal. 226, 215 (2004)

68. S. X.Yu, Ye, J. Power Sources 172, 133 (2007)

69. A.K. Shukla, M.K. Ravikumar, A. Roy, S.R. Barman, D.D. Sarma, A.S. Aric`o, V. Antonucci, L. Pino, N. Giordano, J. Electrochem. Soc. 141, 1517 (1994)

70. S. An, J.-H. Park, Ch-H. Shin, J. Joo, E. Ramsaamy, J. Hwang, J. Lee, Carbon 49, 1108 (2011)

71. J. Parmentier, S. Saadhallah, M. Reda, P. Gibot, M. Roux, L. Vidal, C. Vix-Guteri, J. Patarin, J. Phys. Chem. Solid. 65, 139 (2004)

72. A. Cabiac, T. Cacciaguerra, P. Trens, R. Durand, G. Delahay, A. Medevielle, D. Plee, B. Coq App. Catal. A. 340, 229 (2008)

73. K.P. Gierszal, T.-W. Kim, R. Ryoo, M. Jaroniec, J. Phys. Chem. B 109, 23263 (2005)

74. K.P. Gierszal, M. Jaroniec, T.-W. Kim, J. Kim, R. Ryoo, New J. Chem. 32, 981 (2008)

75. X. Li, F. Forouzandeh, T. Furstenhaupt, D. Banham, F. Feng, S. Ye, D.Y. Kwok, V. Briss, Carbon 127, 707 (2018)

76. Ch.H. Kim, D.-K. Lee, T.J. Pinnavaia, Langmuir 20, 5157 (2004)

77. S.H. Joo, Ch Pak, D.J. You, S.A. Lee, H.Y. Lee, J.M. Kim, H. Chang, D. Seung, Electrochim. Acta 52, 1618 (2006)

78. Y. Nakagawa, K. Takada, M. Tamura, K. Tomishige, ACS Catal. 4, 2718 (2014)

79. N.S. Biradar, A.A. Hengne, S.N. Birajdar, R. Swami, Ch.V. Rode, Org. Res. Dev. 18, 1434 (2014)

80. A. O Driscoll, J.J. Leahy, T. Curtin, Catal. Today, 279, 194 (2017)

81. P. Panagiotopoulou, D.G. Vlachos, Appl. Catal. A 480, 17 (2014)

82. L. Liu, H. Lou, M. Chen, Appl. Catal. A 550, 1 (2018)

83. X. Hu, R.J.M. Westerhof, L. Wu, D. Dong, Ch-Z. Li, Green Chem. 17, 219 ( (2015), )

84. P. Makowski, R.D. Cakan, M. Antonietti, F. Goettmann, M.-M. Titirici, Chem. Commun. 8, 999 (2008)

85. J. Zhu, Y. Jia, M. Li, M. Lu, J. Zhu, Ind. Eng. Chem. Res. 52, 1224 (2013)

86. M.L. Toebes, F.F. Prinsloo, J.H. Bitter, A. Jos, K.P. vande Dillen, Jong J. Catal. 214, 78 (2003) 